\title{
Electrical Tomography: A Review of Configurations, and Application to Fibre Flow Suspensions Characterisation
}

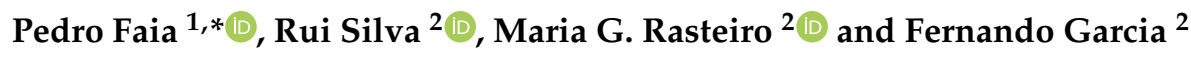 \\ 1 Department of Electric and Computers Engineering, Faculty of Sciences and Technology, CEMMPRE, \\ University of Coimbra, 3030-290 Coimbra, Portugal \\ 2 Department of Chemical Engineering, Faculty of Sciences and Technology, CIEPQPF, University of Coimbra, \\ 3030-790 Coimbra, Portugal; ruiccsilva@yahoo.com (R.S.); mgr@eq.uc.pt (M.G.R.); fgarcia@eq.uc.pt (F.G.) \\ * Correspondence: faia@deec.uc.pt; Tel.: +351-239-796-200
}

Received: 6 March 2020; Accepted: 19 March 2020; Published: 30 March 2020

check for updates

Featured Application: The depicted tomographic technique, Electrical Tomography, is a non-invasive and remotely operated imaging system that makes it possible to obtain valuable information about multiphase system flow behaviours: The 2D/3D images obtained are of extreme importance not only in the development of prediction models, but also in their real-time monitoring and control. It has high applicability in several industrial environments, such as chemistry, paper, petroleum, water and wastes industries, just to mention some.

\begin{abstract}
Understanding the behaviour of suspension flows continues to be a subject of great interest considering its industrial relevance, regardless of the long time and effort dedicated to it by the scientific and industrial communities. Information about several flow characteristics, such as flow regimen, relative velocity between phases, and spatial distribution of the phases, are essential for the development of exact models for description of processes involving pulp suspension. Among the diverse non-invasive techniques for flow characterisation that have been reported in the literature for obtaining experimental data about suspension flow in different processes, Electrical Tomography is one of the most interesting, since it presents perhaps the best compromise among cost, portability, and, above all, safety of handling (indeed there is no need to use radiation, which requires special care when using it). In this paper, a brief review and comparison between existing technologies for pulp suspension flow monitoring will be presented, together with their strengths and weaknesses. Emphasis is given to Electrical Tomography, because it offers the above-mentioned compromise and thus was the strategy adopted by the authors to characterise different flow processes (solid-liquid, liquid-liquid, fibres, etc.). The produced portable EIT system is described, and examples of results of its use for pulp suspension flow characterisation are reported and discussed.
\end{abstract}

Keywords: fibre suspensions flow; electrical tomography; complex flows; disperse phase distribution; velocity evaluation

\section{Introduction}

For the obtention of tomographic information, different measurement methods can be followed with respect to the sensing procedure: intrusive, non-intrusive, invasive, and non-invasive. The choice of an approach is the result of a wise choice made by the users. By 'invasive' is meant that the sensor surface is parallel to the inner zone of the wall of the channel under study, while the term 'intrusive' is used when the sensor itself interacts with the flow being visualised. In opposition non-invasive refers to cases in which the sensor is located on the outside of the wall, without crossing it, while 
non-intrusive indicates cases in which the sensor placement forces the pipe wall to be crossed, but without disturbing the flow under inspection [1]. In recent years, non-invasive procedures have shown a high degree of potential growth in many areas, such as in the chemical industry. When compared with non-intrusive procedures, non-invasive approaches bring benefits to the above-mentioned industry sectors, such as reduction of hazards due to operation of corrosive or radioactive materials, increase in operator safety, etc. Consequently, the non-invasive approach is the imaging strategy most commonly used in process plants.

For hard-field tomography, the case must be considered in which there the measured parameter distribution and the sensitivity of the imaged media in the entire 3D domain are independent of one another [2]; optical, ultrasonic and X-ray are examples of this type of tomography. On the other hand, soft-field tomography refers to cases in which a dependency is found, over the entire volume, between the medium sensitivity and the distribution of the measures of the desired variable [3]. Electrical tomography is the most relevant member of this class, but it presents an important effort regarding the resolution of the inverse problem on the domain under observation.

\section{Pulp Suspensions Imaging Overview}

Pulp suspensions are characterised as being opaque and intricate mixtures that exhibit a quite heterogeneous distribution of fibres under flow, presenting areas in which flocs are present (the term flocs refers to a high concentration of fibres). In addition, along the entire pulp suspension and above certain concentrations, as a function of the observed shear rates, a continuous fibre network forms. For the purposes of process control nowadays, knowledge regarding flow characteristics is essential, once the suspensions have been subjected to high shear forces. The numbers regarding turnover in the pulp and paper industry confirm the importance of this knowledge, as in some countries they exceed the return from other industrial areas, as is the case in Sweden and Canada. Understanding and controlling the manufacturing process enables the development of newer products with a high innovation content. Additionally, obtaining precise values for the velocity profiles associated with a certain shear rate in a pulp suspension is also of high importance for optimising the flow and, consequently, the energy consumption; for the later, however, there is scarce information reported in the literature.

Diverse flow imaging methods, along with particle following velocimetry approaches, have been described in the literature; however, most of these need to be experimentally trained, with data acquisition procedures thus usually lasting quite a long time, and they can typically only be used in non-opaque suspensions. Consequently, it is not possible to use them in pulp suspension flows not belonging to the dilute range. For data collection in pulp suspensions, among the more promising techniques can be found Laser Doppler Anemometry (LDA) (Optomet GmbH, Darmstadt, Germany) [4], Magnetic Resonance Imaging (MRI) (National Institute of Biomedical Imaging and Bioengineering, Bethesda, MD, USA) [5], Ultrasonic Velocity Profiling (UVP) (MET-FLOW S.A., Lausanne, Switzerland) [6], Optical Coherence Tomography (OCT) (PHOENIX TECHNOLOGY GROUP, Pleasanton, CA, USA) [7], Particle Image Velocimetry (PIV) (DANTEC DYNAMICS, Skovlunde, Denmark) [8], X-ray tomography (Department of Physics P.O., University of Jyväskylä, Jyväskylän yliopisto, Finland) [9], Gamma ray densitometry [10], and Electrical Tomography (ET) (PASI SRL, Torino, Italy) [11].

Magnetic Resonance Imaging is a tomographic technology with a high spatial resolution that can supply information regarding the behaviour of the protons present in a system, which are typically contained in the $1 \mathrm{H}$ nuclei of water. MRI can be used, among other applications, to image the motion of water, e.g., expressed in the form of velocity profiles. The major limitations of MRI are the types of particles and the size of the system that can be studied, together with the size, weight and cost of the instrumentation. Only particles containing MR-sensitive nuclei, such as $1 \mathrm{H}$, can be detected $[12,13]$. The maximum diameter of the system is bounded by the inner diameter of the coil in the magnet. Thus, MRI experiments are typically limited to laboratory-scale fluidised beds or pipes with small 
diameters [14]. In summary, the strength of MRI is in studying centimetre-scale systems at spatial resolutions of $\sim 100 \mu \mathrm{m}$. Time-averaged velocity profiles of diluted pulp suspension have been obtained using MRI with fibre concentrations of up to $0.86 \%(w / w)$ [15-20]. However, when compared with LDA and UPV, MRI presents some disadvantages, such as longer observation times and equipment cost. Arola et al. [20] were able, to gather information about the suspension velocity profiles of $0.5 \%$ fibres diluted in water $(\mathrm{w} / \mathrm{w})$ using MRI in a matter of milliseconds. Also using MRI, under steady flow conditions, and for pulp suspensions with concentrations above 3\% (w/w), Seymour et al. [21] were able to measure time-averaged velocity profiles. Despite these lab trials that have taken place around the world, wide MRI usage in industry is not expected in the coming years.

Laser Doppler Anemometry (LDA) is an optical technique used to investigate velocity and turbulence in gas, liquid, and mixed fluids, among other applications [22]. The basic idea underlying LDA is to measure the velocity of tiny particles transported by the flow. If these particles are small enough, their velocity is assumed to be that of the liquid stream, and LDA thus provides a measure of the local instantaneous velocity, the mean velocity, and the turbulent quantities. Laser anemometers offer unique advantages in comparison with other fluid flow instrumentation [22]: (1) Non-contact optical measurement-LDA probes the flow with focused laser beams and can determine the velocity without disturbing the flow; (2) Well-defined directional response- the quantity measured by LDA is the projection of the velocity vector in the measuring direction defined by the optical system; (3) High spatial and temporal resolution - the optics of the laser anemometer are able to define a very small measuring volume, thus providing good spatial resolution and allowing for local measurement of velocity; (4) Multi-component and multi-directional measurements-combinations of laser anemometer systems with component separation based on colour, polarisation or frequency shift allow one-, two-, or three-component LDA systems to be put together based on common optical modules. So indeed, LDA has a lot of advantages, but some compromise has to be made when selecting and setting up laser anemometer systems; indeed, among the disadvantages of LDA are the high cost of the equipment, the need for a transparent flow through which the light beams can pass (usually corresponding to a low concentration of the disperse phase), and the fact that they do not give continuous velocity signals. Nevertheless, the special properties of the gas lasers (such as high energy, spatial and temporal coherence, and stability) make this method applicable for solving a large number of problems. LDA has been used to examine low concentrated pulp suspensions containing air bulbs by Ek et al. [23]; carry out a study of $0.5 \%$ fibre suspensions in water $(\mathrm{w} / \mathrm{w})$ by Kerekes et al. [24]; study pulp suspension flow at concentrations between 1.2 and $12 \mathrm{~g}$ per litre of water, using transparent model systems, based on the refractive index matching of the liquid and the fibres by Steen $[25,26]$; and, using the same model system presented by Steen, performing measurements in a stirred tank with fibre concentrations in the range 3-20\% (w/w) by both Andersson et al. [27] and by Pettersson and Rasmussen [28,29].

Since Acoustic Doppler devices achieved easy handling and low cost, their use has experienced a high rate of growth in recent years. Doppler effect-based echography equipment allows the fabrication of systems that are able to measure velocity profiles nearly instantaneously [30]. The Ultrasonic Velocity Profile (UVP) technique was initially limited to the inspection of non-transparent fluids [31], and to measurements through pipe walls in small-scale systems [32]. In the UVP technique, a probe, acting firstly as an emitter, produces an ultrasound signal that travels along a certain direction, usually the suspension direction, and later, acting as a receiver, senses the reflexions originated by particles present in the suspension; the time, as well as the Doppler frequency shift between emission and reflections, is measured. Using the value of sound velocity in the fluid, the distance and velocity of the particles originating the echoes (the reflexions), along the suspension direction, are calculated. UVP was originally established for 1D measurements; however, promising $2 \mathrm{D}$ velocity profiles have recently been published [30]. The UVP technique has been used extensively in recent years to obtain instantaneous velocity profiles, but very few studies on pulp suspensions can be found in the literature. Hirsimäki [33] used an early ultrasonic profiling technique to obtain radial velocity profiles in pulp suspensions with concentrations up to $1 \%(\mathrm{w} / \mathrm{w})$. Karema and his co-workers $[34,35]$ 
characterised velocity fluctuations and studied paper formation by fluidisation and reflocculation of wood pulp suspensions with concentrations up to $1 \%(\mathrm{w} / \mathrm{w})$ using UVP. Wiklund et al. [31,36] obtained instantaneous velocity profiles in a steady laminar flow of $0.5-3 \%(\mathrm{w} / \mathrm{w})$ and later up to $7.8 \%(\mathrm{w} / \mathrm{w})$ cellulose pulp suspensions using UVP. Claesson and her co-workers [37] used UVP to characterise pulp fibre suspensions through a sudden expansion at two different concentrations, $1.8 \%$ and $2.8 \%$ $(\mathrm{w} / \mathrm{w})$, and at diverse velocities, from 1 up to $2.2 \mathrm{~m} / \mathrm{s}$ : They were able to instantaneously obtain velocity profiles as well as axial real mean square velocity fluctuations in the evaluated semi-concentrated flows just after the expansion. To enable improved flow information for higher concentrations, Kotzé et al. [38] tested a pulsed velocimetry-based system to measure velocity profiles of cellulose flows for two different concentrations, $6.9 \%$ and $8 \%(\mathrm{w} / \mathrm{w})$, in three different pipe diameters $(16,22.5$ and $52.8 \mathrm{~mm})$. Also using a pulsed approach, Hanjiang and colleagues [39] obtained velocity profiles of fibre flows in a rectangular channel, and investigated the influence of fibre concentration and Reynolds number on the shape of the measured profiles; they used natural wood fibres (with an average length and diameter of 2.3 and $35 \mu \mathrm{m}$, respectively), and the tests were conducted with varying average flow velocities of between 0.16 and $7.0 \mathrm{~m} / \mathrm{s}$, and different fibres concentrations between $0.05 \%$ and $1.0 \%(\mathrm{w} / \mathrm{w})$.

Optical Coherence Tomography (OCT) is a recent technique that allows the internal structure and the motion behaviour of opaque suspensions to be measured simultaneously and with high spatial resolution. Depending on the technology used in the OCT construction, the axial scan rates can go up to hundreds of $\mathrm{kHz}$ [7]. OCT operating modus is quite similar to ultrasound imaging, with the main difference being its operating frequency; while OCT operates in the light frequency band, ultrasound uses the sound band. The depth of the obtained images depends to a large extent on the optical properties of the domain; consequently, its distance from the wall can vary between micrometres and some millimetres [40]. This technique was used by Kataja et al. [41] to characterise the boundary layer of micro-fibrillated cellulose flowing in a straight pipe, for consistencies in the range $0.4-1.6 \%(\mathrm{w} / \mathrm{w})$. However, in the case of ultrasound systems, which usually operate in the MHz frequency band, the waves are readily transmitted to the domain, making it easy to obtain images of significant depth: nevertheless, at those frequencies they can also suffer from high attenuation, limiting then the used frequencies. Furthermore, it is much more difficult to focus sound waves than light waves [42].

Particle Image Velocimetry (PIV) is a laser measuring technique, as is LDA, which measures the velocity by means of tracking tracers in the suspension. However, PIV measures the velocity by cross-correlation algorithms, allowing the velocity 3D-components or the velocity gradient tensor in the measuring plane to be obtained [43]. PIV has the advantage of not being a single point measurement technique, and consequently is attracting increasing interest in the evaluation of complex and turbulent flows. The main disadvantage of this technique is the limitation it presents when more opaque domains are the object of visualisation. Sorokin and his co-researchers used PIV to estimate sets of 2D pulp flow velocity vectors [8]. With recent improvements in digital imaging, PIV also makes it possible to obtain information about the orientation of the fibres, as in Fan et al. [44]. In the experiments, they used synthetic fibres with diverse aspect ratios $(18.8,25.0$ and 37.5) in two different concentrations, $0.1 \%$ and $0.2 \%$. They obtained important information about the influence of the impeller speed, fibre aspect ratio and concentration on the field velocity and orientation. Fock and colleagues [45] used PIV combined with LDA to further understand the mechanisms of plug flow and near-wall behaviour in fibre flow through a pipe at concentrations of up to $4.7 \%(\mathrm{w} / \mathrm{w})$ with a flow rate of $0.003 \mathrm{~m}^{3} / \mathrm{s}$; the obtained results showed that the pulp suspension flow in the plug regime can be very inhomogeneous in the region near the pipe wall, particularly at low flow velocities, becoming more structured with an increase in flow velocity.

Among the difficult field techniques, $\mathrm{X}$-ray computed tomography is the safest one; it has excellent spatial resolution and image reconstruction is easy, but due to the source and construction of detection components, its operation is slow [9]. X-ray usage for fibre flow characterisation has rarely been reported in the literature; however, flash $\mathrm{X}$-ray radiography has been used to characterise pulp flows similar to those that can be found in the paper industry. Flash X-ray is an X-ray radiography method 
that uses an intense burst of radiation that is produced for a short time period, making it possible to capture images of high-speed events that are hidden by, for instance, dust, smoke or light [9]. Farrington used Flash X-ray [46] in order to image tungsten fibres with a median diameter of $25 \mu \mathrm{m}$ (acting as tracers) in a wood fibre suspension with a concentration of $2 \%(w / w)$. He was able to asses fibre mass distribution, confirming that this technique could be used for pulp flow characterisation. Later, Heidel and co-workers $[47,48]$ used Flash X-ray to characterise and quantify gas behaviour in several suspensions containing fibres; they used it to visualise air flow in diverse systems, particularly in one composed of air/water/wood fibres, with fibre consistencies of $0.5 \%, 1 \%$ and $1.5 \%(\mathrm{w} / \mathrm{w})$, while varying the average injection velocity of the air between 0.1 and $12.5 \mathrm{~cm} / \mathrm{s}$ and maintaining the remaining mixture injection velocity at a constant rate in the pipe.

Gamma ray has also been tried for inspection of pulp suspensions, particularly for acquiring density/void fraction information [49]. Gamma ray densitometry [10] uses Gamma radiation, which is an electromagnetic radiation similar to X-rays, but which is produced by the natural decay of radionuclides. It is reliable for the determination of densities, it is inexpensive, and it is portable; however, it does not allow local information to be obtained (the collimated beam of the Gamma ray produces a line averaged value and local information in points of the chord cannot be obtained) [50]. Also, Gamma rays' intensities in the domain are quite dependent on the thickness of the pipe wall, and their accuracy depends on the radiation reaching the detector, which is affected by the material and thickness of the pipe wall [50]. Xie et al. [51] combined Flash X-ray tomography with Gamma ray densitometry to study flow structures, gas holdup, and the geometry of the gas bubbles in a gas-pulp-liquid system; in their work, low-fibre suspension consistencies were used-up to $1.5 \%$ $(\mathrm{w} / \mathrm{w})$ - and superficial flow velocities of gas and liquid/pulp suspensions in the ranges $0-26$ and 21-51 $\mathrm{cm} / \mathrm{s}$, respectively.

The study of larger-scale systems can be addressed by Electrical Impedance Tomography (EIT), which makes it possible to obtain good images of the distribution of gas-liquid-solid mixtures [52]. Low cost, portability and ease of upscaling are some of the advantages of EIT. However, its spatial resolution is lower than that of other tomographic techniques. This is mainly due to the number of matchless conductivity measurements that can be obtained, which is strongly tied to the current injection-voltage acquisition system protocol and to the number of measuring electrodes [53]. The reconstruction procedure is poorly conditioned and non-linear [53]. Consequently, the amount of fibres or particles present in the suspension cannot be precisely calculated, with the obtained tomographic images representing the distribution of variation in conductance/impedance, rather than absolute values. To maximise the EIT conductivity distribution images resulting from the reconstruction process, the conductivity difference between the two phases to be distinguished should be large, with larger conductivity differences providing better distinguishability and measurement accuracy. In the pulp and paper industry, EIT has recently been used in applications including the measurement of mixing quality, flow uniformity, and velocity, just to mention a few [11,54-56].

\section{Electrical Tomography Principles and Applications}

\subsection{Electrical Tomography}

Due to its low cost, portability and handling safety (because no harmful radiation is used), Electrical Impedance Tomography exhibits strong potential for use in several academic/industrial areas. It has a simple setup, and offers quick and easy operation, making it robust enough to be used in many industrial situations. As has already been mentioned, its main drawback is its low spatial resolution, which is usually confined to between $6 \%$ and $20 \%$ of the pipe radius [53]. However, with the development of more advanced algorithms for inverse problem resolution, there have been significant improvements with respect to increasing the achievable spatial resolution. With Electrical Tomography, both quantitative and qualitative data regarding multiphase systems can be obtained for modelling purposes. 
Non-invasive collection of data by electrical tomography enables the reconstruction of cross-section images, providing material distribution profiles in a suspension flowing through a pipe, or information about possible transient events taking place. The resulting conclusions can then be used in processes redesign or control. In some cases of the real-time imaging of industrial processes, electrical tomography is the most appealing technique, due to its simplicity and low cost. Electrical tomography can be divided in two procedures: Electrical Impedance Tomography, EIT (note that Electrical Resistance Tomography, ERT, can be seen has a particular case of EIT), and Electrical Capacitance Tomography, ECT. They both allow the generation of images that reflect the variation of the conductivity/resistivity or permittivity along the under-observation domain; due to its simplicity of implementation, ERT, which is ideal for pure resistive domains, is the most used [57].

When systems are heterogeneous, and composed of materials possessing diverse electrical characteristics, EIT and ECT can be used in the observation of changing processes; pneumatic and hydraulic transportation, mixing, cyclones, and fluidised beds are only some examples. The number of images generated by time interval is variable: it depends mainly on the electronics and structure of the acquisition system, on the measurement protocol used to gather the data, and on the reconstruction algorithm used for image building. Typically, increasing the data gathering speed would make it possible to obtain data about faster process, but consequently with a higher noise level and reduced image quality of the distribution profiles. Electrical tomography can be used on-line or off-line. For on-line measurements, poor spatial resolution images are usually obtained, consequence of the need to use either fast acquisition procedures or data processing algorithms that only require a short time. Conversely, for off-line measurements, the acquisition times can be customised to adapt to the changes taking place in the flow process, while the processing algorithms can be optimised to the highest spatial resolution possible, because the processing time can be longer.

In addition to the reconstruction algorithm used, other features determine the quality of the obtained images, mainly physical ones, such as the number of electrodes, electrode diameter and material, spacing between them, etc. Because in some applications the aim is to obtain information about average quantities, such as void fraction or mean velocity, the developed model's validation requires images with high spatial resolution.

In the following sections, the authors start by addressing the basic principles and operation of Electrical Impedance Tomography, followed by a short review of the use of Electrical Tomography for industrial/process monitoring purposes, with an emphasis on the pulp and paper area. Finally, a succinct description of the authors' approach to developing high spatial resolution portable EIT equipment is presented; the authors' EIT system makes it possible not only to inspect media with high conductivity, as are typically found in industry, as well as using a reconstruction procedure that makes it possible to obtain sharper impedance distribution images. The results obtained with this new system using fibre suspensions on a laboratory level pilot rig will be presented. Finally, the incoming challenges for Electrical Tomography are addressed.

\subsection{Fundamentals of Electrical Tomography}

Maxwell [58] was the first to present a theoretical model connecting the permittivity/conductivity of a two-phase mixture to the volume fraction of one the compounds present in the mixture dispersed in the second. For his calculations, he used small spheres of a certain material possessing equal but small size when compared with the distance between them; additionally, he presumed that in a certain domain, the spheres were distributed in a uniform way along the continuous phase of a second material, and that consequently their presence disturbs a homogeneous electrical field passing through the domain. Extrapolating, by measuring quantities such as conductance/capacitance, between a pair of electrodes placed around the pipe wall, the particle distribution inside that pipe can be obtained by means of the distribution of the electrical properties and through the usage of an adequate mathematic procedure: this mathematic algorithm links the measurements with the particle distribution. The relation between the homogeneity of a multiphase flow and this idea has been 
used for some time to observe different systems, such as liquid-solid and gas-liquid. For instance, to quantify localised amounts of solids in a gas flow, small capacitive probes have been used $[59,60]$. In the gas and oil industry, similar probes have been used with analogous objectives [61]. Typically, in electrical tomography, the excitation sources, either a voltage or a current source, operate at low frequencies (below $5 \mathrm{MHz}$ ). Consequently, the systems in which these types of sources are used are described by the governing equations of the electrostatic field. When the flux (or current) lines encounter an interface with different permittivity or conductivity, it gets deflected. In order to allow the mining of the maximum amount of information about the process, the electrodes are usually mounted over the periphery of the pipe at intervals of equal distance. In the case of capacitive systems, the measuring electrodes are installed outside of the process vessel or pipe which is made of a dielectric material (i.e., they are mounted in a non-invasive way). Furthermore, the electrode area must be high, making it possible to detect a large range of capacitance changes. For system measuring Resistivity changes, the sensing electrodes are quite small when compared to those used in capacitive systems. They will typically be in contact with the flow under inspection, mounted level along the inner side of the pipe wall.

\subsubsection{Capacitance Tomography}

The purpose of ECT it to obtain a reconstruction of the dielectric characteristics of a system, using measures of capacitance taken between all existing electrode pairs. In Figure 1, a schematic of a cross-section representation of an ECT system possessing eight electrodes can be seen. If the pipe where the suspension is flowing is made of a conductive material, then the electrodes are internally mounted. If, conversely, the pipe is made of an insulating material, then the electrodes will be located externally. External electrodes are easily conceived, assembled and maintained; additionally, once they have been directly exposed to extreme turbulence, pressures and temperatures, their electrical behaviour remains unchanged for a longer time (contamination by the materials flowing in the tube is not encountered, either). Their greatest disadvantage is that they possess non-linear characteristics; however, the use of adequate correction factors allows them to display almost linear characteristics. The design of internal electrodes is harder and more intricate, because they are exposed as described above to extreme conditions, and may also suffer from corrosion; however, the capacitance changes observed with internal electrodes can be assumed to be directly proportional to the permittivity changes occurring inside the pipe. In ECT, all electrodes are stimulated, one at a time, and all pairs of capacitances between the stimulated electrode and the remaining ones are measured; for an $N$ electrodes system, this produces $N(N-1) / 2$ independent measurements, once capacitance $C_{i, j}=C_{j, i}$ and $C_{i, i}$, i.e., the self-capacitance, are all ignored. In summary, the measuring protocol operates in a similar way to the source-detector movement in computerised tomography in medical imaging; in ECT, the electrical field rotates around the pipe cross-section in steps, with a step angle of $360^{\circ} / \mathrm{N}$. The number $N$ of electrodes is chosen based on a balance between the desired spatial resolution and acquisition rate of the images (acquisition rates around 100 frames per second are frequent). Maxwell equations make it possible to establish a relationship between the measured capacitances and the actual spatial distribution of the permittivity. In ECT, only one electrode is stimulated at one time, while the remaining are at a virtual earth potential. Consequently, over tall electrode surfaces, the total electric flux is equal to zero. The capacitance measures can be easily influenced by external parasite capacitances (usually greater than the measured ones), such as earth capacitance, because the inter-electrode capacitance is quite small. To prevent this perturbation, electrode shielding is implemented. With respect to the excitation signal frequency, a value of around $1 \mathrm{MHz}$ is common; for this order of magnitude, the signal wavelength is of some hundreds of meters, greatly exceeding the sensor size by many orders of magnitude. In summary, electrostatic field theory can be described by the electrical potential distribution inside the medium under inspection. 


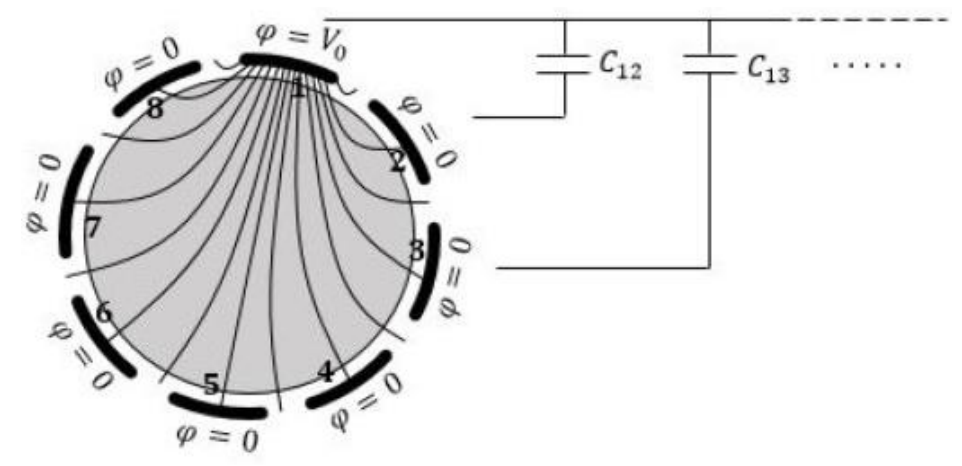

Figure 1. Schematic representation of the measurement principle of an ECT system (reproduced with permission from Hansen et al. [62]).

ERT sensing arrays are less complex than those required by ECT, and the difficulty in their conception arises when the domains present high conductivity $[63,64]$. Consequently, it can be stated that it is quite adequate for the inspection of non-conductive mixtures containing diverse permittivity's. ECT produces low-resolution images, but, when dealing with disperse system flows, offers good accuracy with respect to volume fraction estimation [65]. If in ECT a dual plane system is used, then cross correlating the cross-section average time series enables the evaluation of a particle's velocity [65].

\subsubsection{Resistivity/Impedance Tomography}

The impedance variation distribution of a mixture is the focus of EIT. In EIT, the stimulus used is a sinusoidal electrical signal (current or voltage) with a certain frequency and amplitude. Then, the real and imaginary parts of the impedances are calculated using phase sensitivity measurements $[66,67]$. The resistive component is obtained by an in-phase measurement with the stimulus signal, while the capacitive component is found by a quadrature of phase measurement with the excitation signal [53].

The invention credit for EIT is given to John G. Webster, who reported it in a 1978 publication [68]. Still, the first known experimental application of EIT took place in 1984, for which the authors were Barber and Brown [69]. For investigating processes where the continuous phase is electrically conductive, either ERT or EIT can be used. Specifically, when the inspected medium is purely resistive, then ERT should be used. Similarly to ECT, in ERT/EIT an electrical current is injected through a pair of electrodes that are placed along the boundary of the domain under study. Consequently, the resulting electrical field distribution in the domain will be conditioned by the material distribution within it [70].

The potential voltage differences between all pairs of electrodes placed around the domain perimeter, apart from the pair used for exciting the domain, are measured, and used to nourish a non-linear algorithm. This algorithm solves the inverse problem described above and permits the calculation of the unknown conductivity/resistivity distribution along the domain. However, the procedure only ends when all electrodes are used for stimulating the domain, and thus a full cycle has as many projections as the number of electrodes comprising the sensing system (see Figure 2) [71]. Therefore, in summary, for estimating the material distribution in the domain, an electrical field distribution characterisation is used. In ERT, which is easier to implement [67,72], the aim is to obtain a conductivity/resistivity distribution along the domain. The working principle used in ERT to obtain the data related to a full set of projections, is identical to that described for EIT (requiring the use of all electrodes to introduce the electrical excitation signal in the domain, and to measure all the potential voltage differences between the remaining ones). The single difference is that in ERT, the electrical signal used as stimulus is a continuous current. Therefore, for EIT, the data acquisition cycle only ends when a full rotation of the applied electric field excitation is complete. Consequently, for both EIT and ERT, if the sensing system is composed of $N$ electrodes, the number of independent differential voltage measurements is $N(N-3) / 2$. Typical values for the frequencies used in EIT systems lie in the range $20-150 \mathrm{kHz}$, so quasi-static conditions can be assumed when a full rotation data is 
acquired. In recent years, efforts have been directed towards the development of acquisition systems and to the conception of sensors (materials used in their fabrication, dimensions and location of the sensors, etc.). The use of a discretised electrode system is restricted to based flows with constant conductance [73]. When large bubbles are present in the fluid, those bubbles can cause electrodes to lose contact with the fluid, and consequently, the obtained images of the conductance distribution will be inconsistent. When faced with this type of suspension, one possible solution would be the use of continuous electrode rings instead of a discrete electrode sensing system [73]. An additional limitation of using discrete electrodes mounted in the pipe wall, the surface of which is parallel to the inner pipe wall (as required by resistive/impedance tomography), is their surface modification over time, particularly when used for evaluation processes that operate under extreme conditions. Temperature is one example of such conditions: temperature changes can dramatically cause fluid conductivity to alter, and consequently fluid temperature compensation mechanisms have to be applied to "correct" current/voltage measurements.
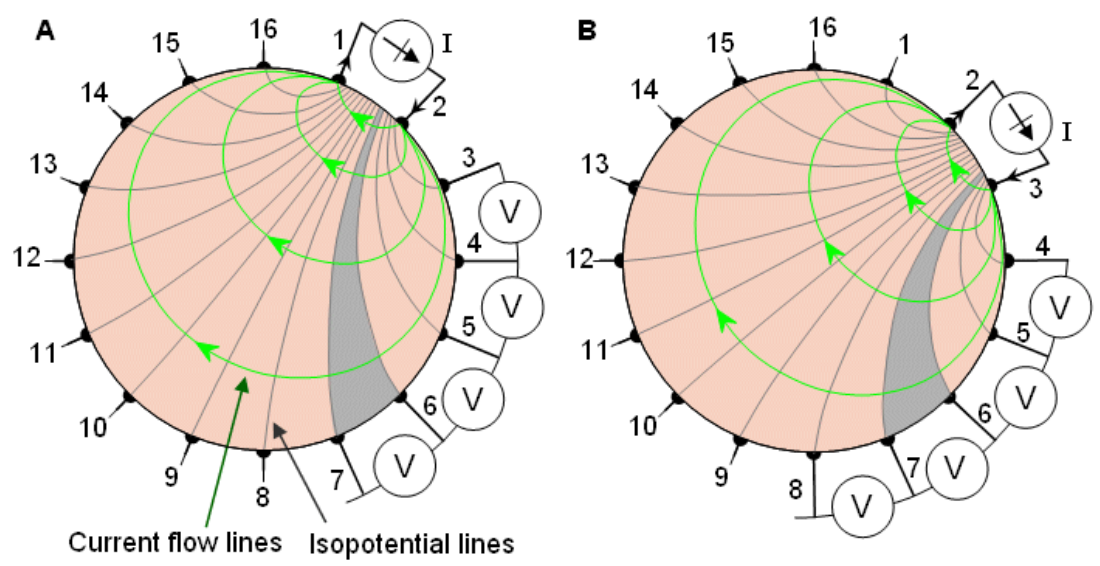

Figure 2. EIT/ERT adjacent injection and measurement protocol for the first (A) and second (B) projections (reproduced with permission from Malmivuo et al. [71]).

Looking now to the evolution of acquisition systems, recent developments in the electronics field have made it possible to achieve frame acquisition speeds of 1000 frames/s [73,74]. In acquisition systems, a good excitation strategy is also vital, and these are typically current-based ones. However, the use of voltage-based excitation strategies, where a voltage-controlled source (typically with low output impedance) combined with an equal width pulse synthesiser, generates a subset of waveforms used for exciting the domain and to implement demodulation, have attracted increasing attention, since they produce less noisy data [73]. The use of voltage-controlled sources in the domain excitation causes electrical currents inside the domain that increase with the increase in suspension fluid conductivity; this fact is very important for highly conductive suspensions, above $2 \mathrm{~S} / \mathrm{m}$, which are quite typical of industrial processes $[67,74]$.

In addition to the shape and size of the electrodes, and the material from which they are made, when using ERT/EIT, other characteristics must be considered, such as the spacing between electrodes, and for EIT the frequency of the electrical excitation signal. Altogether, these characteristics condition the electric field distribution along the domain and determine the true measuring volume and overall sensitivity $[74,75]$. However, some of the negative effects of these characteristics can be overcome; for instance, the effect of the electrode dimensions can be reduced by using appropriate calibration procedures [76]. EIT, similar to ECT, can also be used to obtain disperse phase velocity by using a pair of data acquisition planes and applying cross-correlation algorithms to the acquired data [77,78]. However, to properly estimate velocity, a minimum frame rate acquisition of 100 frames per second is needed [78]. Demodulation has to be performed through software to overcome the problem of the low settling time of the low-pass filters. 
The way electrodes are combined to introduce the electrical signal into the domain is designated by the Injection Protocol. Thorough studies on injection and measurement protocols or patterns can be found in the literature. The most common strategy is referred to as the four-electrode method, where the signal is injected through some electrodes and the voltages are measured from the remaining electrodes. Different patterns for using this strategy can be found in the literature [79,80]: (a) Adjacent Pattern: where the signal is injected through neighbouring or adjacent electrodes and the resulting electrical potential differences are measured in the remaining pairs of electrodes, in a similar adjacent combination; (b) Opposite Pattern: In this pattern, the signal is injected through opposite electrodes and the electrical potential is measured with respect to one reference electrode adjacent to the injecting electrode. In addition to the above-mentioned strategies, which are the most frequently used, others have been employed, such as the Cross or Diagonal Pattern [80], which is a combination of the two described above, and the Trigonometric or Adaptive Pattern [81].

\subsubsection{Image Reconstruction}

For all electrical tomography techniques, the performed measures are sensitive inside a certain volume; this sensitivity is non-constant along the section of interest contained within the volume, and for any given position inside this section, it is also dependent on the spatial resolution of the physical parameter that is being imaged along the entire section of interest. This non-linear behaviour makes obtaining images for the processes under evaluation quite difficult. All electrical tomographic procedures are governed by analogous partial differential equations, and consequently the reconstruction algorithms used share many similarities. Using the data measured, capacitances and potential voltages differences in ECT and ERT/EIT, respectively, in order to construct an image of the domain under evaluation is called inverse problem solving; conversely, inferring the values that will be measured based on a known image of the domain is called forward problem resolution (it is a question of mapping a set of hypothetical parameters onto a set of experimental measurements). For ECT, forward problem-solving means calculating the capacitance values in the domain for a given dielectric permittivity inside the same domain, and this problem has a unique solution.

With respect to inverse problem solving, aside from being a non-linear problem, it is also ill-posed and ill-conditioned; mathematically speaking, its solution is based on a matrix inversion approach (in ECT, this corresponds to the determination of the material distribution in the domain by means of capacitance data values). Inverse problem solving is also referred to as a reconstruction problem. Mathematically, the objective is to calculate the reciprocal of the operator $\mathrm{F}$, which describes the original distribution of the dielectric characteristics in the domain, $\varepsilon=\varepsilon(r)$, using the measured capacitance data values $C^{*}$ :

$$
\varepsilon(r)=F^{-1}\left[C^{*}\right]
$$

Due to its non-linearity, there is no analytical answer to this reconstruction problem. The number of variables that are unknown is higher than the collected measurements, and consequently an infinite number of solutions exists matching the measured capacitances. The best solution will be the constructed permittivity distribution that best fits the measured capacitance values.

The forward and reconstruction problem solving using EIT follow analogous approaches. In the forward or direct problem, an approximation of the electrical potentials in the boundary of the domain is calculated using the initially estimated conductivity/resistivity distribution. On the other hand, in the reconstruction problem, the conductivity/resistivity distribution in the domain is estimated using the values of the electrical potentials measured along the domain boundary, using an appropriate mathematical algorithm. In the direct problem, and using Maxwell laws [58], a model of the electric field distribution in the domain is constructed. In any case, before addressing the reconstruction problem, it is necessary to calculate the solution for the forward one, which will be performed using a calibration tool. Typically, Finite Element Methods (FEM) are used to solve the forward problem, i.e., to calculate the voltages/capacitances at the boundary of the domains for a known permittivity/conductivity distribution inside the same domain. A sensitivity matrix is obtained from the 
measured capacitances/electrical potential differences values. The direct problem solution obtained is then fed into the inverse problem algorithm, together with the measured capacitances/electrical potential differences values, which calculates the permittivity/conductivity distribution. The interpretation of the observed permittivity/conductivity distribution is carried out using the sensitivity matrix and the measured data along the boundary.

The methodologies used for solving the inverse problem, and consequently to obtain the 2D cross-sections of the domains, can be classified into three categories: linear; non-linear iterative, and heuristic multivariate methods.

- Linear methods (single-step and iterative methods): In this approach, images are generated by simply multiplying the measurements by a pre-calculated matrix, and consequently they are quite fast in terms of computational effort. Among the linear methods, Linear back-projection (LBP) is the most widely used (the used matrix is the transpose of an estimated solution of the forward problem [82]). Nevertheless, they are usually non-iterative methods. Images obtained by means of LBP are low-resolution, heavily smoothed ones, because the transpose of the forward problem solution is a poor estimate of the solution to the inverse problem. To circumvent some of these limitations, an iterative back projection algorithm was proposed by Yang et al. [83]. Linear methods have been improved by including approaches based on ridge-regression and eigenanalysis [84].

- Non-linear methods: in this approach, numerical forward solvers that predict the measurements, together with sensitivity maps that make it possible to calculate the measurement residuals, are used to estimate the image. Then, in a second stage, that image is updated via a non-linear iterative scheme, such as the modified Newton Raphson (NR) [85] or adaptive mesh regenerating techniques [86]. However, because NR can introduce artificial errors into the solution, regularisation procedures, such as the Marquard and the Tikhonov procedures, are applied to obtain a better approximation at each iteration [73]. In cases where direct inverse solution algorithms are used, there is a search for the minimised residual vector, making it possible to obtain images with improved accuracy $[73,74]$. In cases where higher measurement flexibility is desired, non-linear iterative methods are to be recommended; however, the considerable computational effort makes image reconstruction difficult and too slow. Real-time reconstruction of images using non-linear iterative methods is their major drawback, although this may change through a combination of efficient algorithms [87] (Molinari et al. [88] decreased the time needed to reconstruct images using an adaptive mesh grouping method based on a fuzzy genetic algorithm). The increase in computing performance will also help to decrease the time required for image reconstruction. In FEM methods (Finite Element Methods), which are mesh-based reconstruction algorithms, in order to obtain higher-resolution images, mesh optimisation is also of major importance [74].

- Heuristic methods: these methods can be linear or non-linear, and they model empirically the relationship between calibration sets of images and measured ones. Methods making use of multiple linear regressions [89] belong in the linear sub-class. Self-organised maps and artificial neural networks [90] are examples of methods belonging to the non-linear sub-class. Heuristic models also allow the mathematical relations between the measurements and other variables of interest to be obtained [91]. In many cases, image reconstruction in process applications is just an intermediate stage towards the calculus of other variables.

\section{Electrical Tomography Applications}

\subsection{Examples of Applications of Electrical Tomography to Chemical Engineering Processes}

Many and diverse applications of ET can be found in the literature. The authors present a non-exhaustive overview of the applications, media, and purposes that can be found in the literature. 


\subsubsection{Operating Apparatus}

Pipes, storage and mixing systems are among the most common devices described in the literature in which Electrical Tomography has been applied. The possession of multidimensional information about flow features and settings in fluid (either liquids or gases) transportation systems (which would require being able to see inside the pipes) composed of pipes and piping machinery would provide numerous benefits in process design, monitoring and control. This is why the Electrical Tomography applications described in the literature have without doubt focused on diverse pipes situations. Vertical, horizontal, and inclined straight pipes, helical pipes, and so on are Among the various reported configurations that can be found in the literature. When the focus was on vertical straight pipes, diameters between 50 and $125 \mathrm{~mm}$ have been investigated [92,93]. There are more numerous publications of case studies using horizontal straight pipes addressing diverse flow regimes than for any other configuration, with pipe diameter varying in the range 35 to $85 \mathrm{~mm}[74,94-96]$. Reports on studies using ERT in inclined pipes with different inclination angles [97] are scarce; the same can said for its application in studies with helicoidal pipes [70]. In contrast, storage and mixing containers, which can be found in industrial processes in large number, and which vary with respect to their size, purpose, etc., are the second most studied application area using ET. In particular, researchers have focused on stirred containers possessing various baffles and mixing characteristics with diameters in the interval 15-40 cm [98,99], and on plant-scale containers with diameters of around $150 \mathrm{~cm}[100,101]$.

\subsubsection{Type of Media Conditioning}

Diverse media have been studied, independent of the pipe or container characteristics to which the electrical tomography is applied. Aqueous phase-non-conductive particles, aqueous phase-air, and aqueous phase-oil mixtures, among others, are some of the case studies that can be found in the literature. As already mentioned, a high contrast between the phases present in the media is desirable, once electrical tomography measurements use the conductivity differences to distinguish them.

For instance, if ET is used in a medium consisting of non-conductive particles suspended in a conductive liquid phase, which is a highly common situation in industry, it would be expected to provide valuable information about that media flowing behaviour. Many liquid phases have been used in conducted studies: however, in the majority of them, the chosen medium has been some type of brine or water. In particular, water has been used on a large scale as an aqueous phase medium [56]. However, because water itself has low conductivity, it may not be the most suitable liquid phase in some situations; increasing its conductivity by the adding a salt (e.g., $\mathrm{NaCl}, \mathrm{KCl}$ ) can provide better measurement accuracy $[74,96,102-104]$. With respect to the non-conductive phase, several types of particles have been used in the works reported in the literature: among these are studies using glass, nylon or plastic beads [102,105], lava rock [106], quartzite particles [102], sand [107], and silica [108].

Another widely addressed, two-phase system using electrical tomography, and which is common in many processes, is liquid-air medium. In the conducted works, water has generally been used as liquid phase, while air bubbles, distributed through it, act as non-conductive particles [109,110]. However, understanding the aptitude of electrical tomography for detecting the air bubbles and understanding the relation between the size and location of sensing electrodes and the minimum detectable bubble size are still important areas of study, even if there have already been several reports in the literature about these issues.

Two-phase systems in which both phases are liquids with different conductivities also comprise an important research area, particularly when one of them is an oil. In the literature, there are mentions of the use of linseed oil [111], shell sol D70 [97], sunflower oil [108], and paraffin oil [112,113], among others. In the food industry domain, the dairy industry also presents great potential for the application of electrical tomography. Successful application of ET measurements to yogurt using either water or a salted plug of yogurt to obtain the conductivity difference [114,115] has been achieved. In another situation, electrical tomography measurements on whole and skim milk solutions of various concentrations were performed by Sharifi et al. [116]; as the second phase, and to assure the required 
conductivity difference, they used either another milk solution with a different concentration, cream, or water.

\subsubsection{General Applications of ET}

There are many application areas to which electrical tomography can be applied, including phase distribution analysis and mixing quality analysis. When several sensing electrode planes are distributed along the pipe (and where their distance from one another is known), images of the flow behaviour throughout a time period can be obtained for each plane; the cross-section correlation of the images permits the estimation of information related to, for instance, velocity distribution flow rate, multi-dimensional flow monitoring, flow regime identification, and jet mixing investigation.

Additionally, through the cross-correlation of images obtained from consecutive sensing electrode planes for the passage of a conductivity modification (for instance, a second phase in a two-phase system), estimation of flow velocity distribution can be achieved. Several approaches have been used for cross-correlation; the most commonly used have been the best-correlated pixel, in the case of flow rate measurements [117], and pixel-pixel correlation, in the case of velocity distribution measurement [56].

When the measurements needed for electrical tomography application are performed in mixing containers, in a similar way to flow processes, the obtained multidimensional conductivity data can be used in the investigation of various aspects of the mixing system, such as mixing time and mixer performance. These aspects have typically been the ones to which more attention has been devoted, because they are critical for the analysis of diverse processes and reactions in the Chemical Engineering field; in fact, information about mixing time and performance makes it possible to evaluate, for example, the degree of homogeneity $[75,118]$.

Void fraction is also a very important characteristic in multi-phase systems, because it makes it possible to better understand the observed flow patterns. Several investigations have been carried out using electrical tomography for the measurement of gas phase hold-up or void fraction in multiphase mixtures $[119,120]$. A few researchers, taking advantage of the capacity possessed by electrical tomography to detect conductivity changes in the flow, have used it with the aim of monitoring diverse precipitation processes [101,121]. Electrical tomography has also been used in other domains, such as in the identification of faults or damage, and stresses and pathologies; examples of the latter have been reported by Sharifi et al. [116], who applied ERT for the detection of milk mixing process pathologies, and by Vlaev et al. [122], who used ET to detect and filter cake pathologies in solid-liquid filtration.

\subsection{Applications of ET in the Pulp Suspensions Domain}

As already mentioned, due to low cost and ease of transport, and despite their low resolution in comparison with other tomographic techniques, ET has been used in pulp suspensions analysis. In the next paragraphs, a review of some of applications found in the literature is carried out.

Yenjaichon et al. [123] defined a gas mixing index derived from the standard deviation of cross-sectional local gas holdup obtained from electrical resistance tomography and used it to investigate the dispersion of gas into pulp-suspension horizontal flow downstream of $90^{\circ}$ tees as affected by the fibre mass concentrations in the range $0-3.0 \%(\mathrm{w} / \mathrm{w})$, superficial liquid/pulp velocities $(0.5-5.0 \mathrm{~m} / \mathrm{s})$ and superficial gas velocities $(0.11-0.44 \mathrm{~m} / \mathrm{s})$. The mixing in the turbulent flow of dilute pulp suspensions was similar to that for water but differed significantly at higher suspension concentrations. Mixing worsened with increasing fibre mass concentration for the bubble flow regime, which was likely due to a dense plug of fibres in the core of the pipe causing bubbles to congregate near the wall. When buoyancy was significant, gas uniformity improved with increasing pulp concentration, since robust fibre networks caused liquid/pulp slugs to flow at the top of the pipe, whereas stratified flow was approached at lower concentrations. Mixing was less dependent on superficial liquid/pulp velocity at higher pulp concentrations, due to there being less variation in flow regimes. 
Using the same modified mixing index and electrical resistance tomography, Yenjaichon et al. [54] pursued their research and presented results about the quality of liquid mixing into the mainstream for an in-line mechanical mixer. The experiments were conducted in a pilot-scale flow loop facility, consisting of two $4000 \mathrm{~L}$ tanks, a $40 \mathrm{HP}$ centrifugal pump and a $40 \mathrm{~m}$ length of $76.2 \mathrm{~mm}$ PVC pipe; the acrylic test section, $1.88 \mathrm{~m}$ in length, included eight ERT sensor planes spaced at $250 \mathrm{~mm}$ intervals. The first sensor plane was located $67 \mathrm{~mm}$ upstream of the side-stream injection, and the impeller was $104 \mathrm{~mm}$ downstream of the injection point. The mechanical mixer system consisted of a $0.5 \mathrm{HP}$ motor, a $1.41 \mathrm{~N} \cdot \mathrm{m}$ rotating torque sensor with an integral optical encoder, a $12.7-\mathrm{mm}$-diameter stainless steel shaft, and a 63.5-mm-wide octagonal impeller with a 38.1-mm-diameter hole in the middle to prevent excessive force on the impeller when it was perpendicular to the flow. In the experiments were used: aqueous pulp suspensions with concentrations in the range $0-3.0 \%(\mathrm{w} / \mathrm{w})$; jet velocities ranging from 3.8 to $12.6 \mathrm{~m} / \mathrm{s}$; main-stream velocities in between $0.5-3.0 \mathrm{~m} / \mathrm{s}$; rotational speeds of 0 to $800 \mathrm{rpm}$. Some examples of images obtained in this study are presented in Figure 3. It was observed for all evaluated pulp concentrations that the mixing quality diminished when the jet was far from the wall of the pipe. For higher impeller speeds, the effect of the water flow on the residence time of the mixing was significant. When using the impeller, the quality of the mixed pulp suspensions improved noticeably; it was found that the quality was analogous to the situation in which water flow approaches a turbulent regime, but with a much smaller main-stream velocity needed for the mixing when compared to the case where only the tee mixer is used. For higher pulp concentrations, however, the supplied energy to the process, even when the highest main-stream velocity and impeller speed were used, was not enough to equal the turbulence level attained in water. Improvements on the mixing quality were observed to occur in the high-shear zone around the impeller when the impeller speed increased; however, it was simultaneously observed that turbulence decayed rapidly downstream due also to reflocculation.

ERT was used by Kourunen et al. [124] to evaluate the performance of a pilot-scale medium-consistency mixer: Additionally, they compared the obtained results with those from temperature measurements. A $10 \%(\mathrm{wt} / \mathrm{wt})$ softwood pulp suspension mixture, flowing at a mean velocity of $\sim 2.1 \mathrm{~m} / \mathrm{s}$, was again quantified based on the mixing index (quotient between the standard deviation of the conductivity distribution and the mean value of the distribution of an image data set). When a tracer was used (air, steam or cold water) and added to the mixture, and by the use of ERT, the observed mixing quality decreases; however, when the mixer was on, the mixing quality was enhanced, and the observed results were quite similar to those obtained from temperature profiling. Nevertheless, having performed some trials, the results were not totally conclusive.

Based on Electrical Impedance Tomography (EIT), Zhou et al. [125] described a low-cost system to measure the consistency profile in pulp flow. A 16-electrode sensor was used to acquire the cross-sectional data. Data acquisition was controlled by a single-chip computer, which received commands from a host computer, initialised hardware, acquired data and sent them to the host computer via a serial port. All the measurement parameters such as the amplitude and frequency of the injected current could be set by software in host computer. The consistency profile images were reconstructed by the modified Newton-Raphson algorithm. EIT system was used to measure pulp suspensions in a shallow flow. Experiments were conducted on a laboratory-scale flow rig that was constructed to simulate the spreading of pulp from a headbox. EIT measurements were acquired from a set of linear array electrodes flush-mounted to the base of the slice opening located at the bottom of the headbox. The EIT measurements were derived from a 'sliding' measurement protocol that involved selectively scanning subsets of the electrode array while using the standard adjacent measurement protocol. The results of the experiments showed that EIT could resolve pulp consistency down to $0.1 \%$ $(\mathrm{wt} / \mathrm{wt})$ with a spatial resolution that was less than $1 \mathrm{~cm}$. 
a.

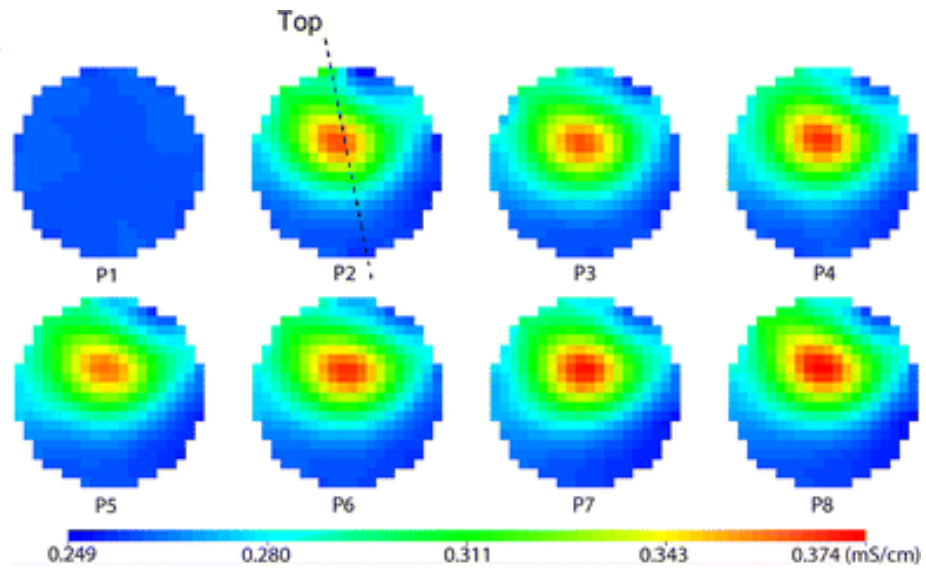

b.

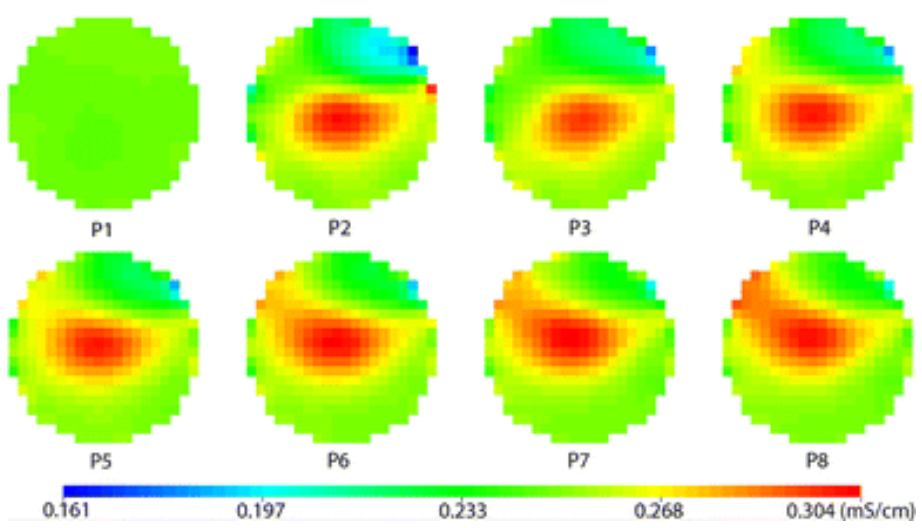

Figure 3. Softwood pulp suspension electrical tomography for $\mathrm{Up}=0.5 \mathrm{~m} / \mathrm{s}$ (main stream velocity), $\mathrm{Cm}$ $=2.0 \%$ and $\mathrm{Dr}=0.05$ (the injection tube length-to-diameter ratio), using sequential sensing planes (the location of the planes P1-P8 is from left to right in the flow direction, with P1 located before the injection point and the impeller), with (a) $\mathrm{R}=12.2$ (velocity ratio between main stream velocity and jet velocity) and $\mathrm{N}=403 \mathrm{rpm}$ (rotation speed); (b) $\mathrm{R}=16.5$ and $\mathrm{N}=410 \mathrm{rpm}$. In all the images the injected tracer is represented by the high-conductivity region in red and yellow, whereas the mainstream is represented by the low conductivity blue region. (Reproduced with permission from Yenjaichon et al. [54]).

Vlaev et al. [55] studied the ability to create uniform liquor flow in the counter-current zones of a lab-scale continuous digester using a $40 \mathrm{~L}$ model digester equipped with an eight-plane electrical resistance tomography (ERT) sensor array. Conductively tagged fluid flows were visualised moving through a stationary bed of uncooked wood chips for a range of process flow conditions. Tests were conducted for a range of flow conditions $\left(Q_{A} / Q_{R}=0.5\right.$ to 2.0, with $Q_{A}$ being the axial up flow and $Q_{R}$ being the radial or screen-circulation flow) and liquor fluxes ( $Q_{A}$ and $Q_{R}$ to a maximum of $\left.250 \mathrm{~mL} / \mathrm{s}\right)$. To create distinct concentration zones within a chip column with liquid flows configured as in a continuous digester the ratio between the axial up flowing liquor and the radial down comer-screen liquor circulation should be less than or equal to one. As the intensity of the down comer screen flow, $Q_{R}$, increased, the zones became more distinct as more of the rising liquor was diluted and withdrawn prior to exiting the screens. While the intensity of the circulation flow does not significantly alter the uniformity of the zones when $Q_{A} \leq Q_{R}$, it may be significant in determining the radial uniformity of temperature and liquor concentration in an operating digester. The flow conditions modelled for two industrial digesters showed formation of distinct and uniform zones above and below the screen region, indicating that they were operating with potential good uniformity.

Ruzinsky et al. [126] investigated digesters (digesters for chemical pulping are pressurised reactors that create a flow of process liquors relative to wood chips to delignify the wood and produce kraft 
pulp) and observed that as their size increased to accommodate higher production rates, operating problems related to liquor flow increased (as defined by the authors, liquor flow is the relative flow of liquor through the chip mass). Among the different problems, they found decreased counter current washing efficiency, accelerated corrosion, increased pulp non-uniformity and problems with chip movement. In a column arranged to operate as the wash section of a continuous digester, they used ERT to obtain images of the flow properties. For the tests carried, the packed bed was modelled using HDPE pellets of high-density polyethylene, having diameters of around $4.44 \mathrm{~mm}$ and thickness of around $1.91 \mathrm{~mm}$. Regarding the liquid phase, a split between liquor flows was made to obtain ratios of axial (up flow in all cases), $Q_{A}$, to radial flow, $Q_{R}$, of $0.5,1.0$ and 2.0. The tests with wood chips showed that liquid flow adjacent to the vessel wall was significantly greater than at the centre of the vessel. This difference was not as significant for the HDPE disks for which the wall-affected zone is much smaller and below the spatial resolution of the ERT system. When a tracer was isokinetically added, ERT reconstructed images below the down comer showed that the entire trace leaved the down comer when $Q_{R}$ was twice the value of $Q_{A}$; nevertheless, for a 1:1 flow ratio, some tracer was detected above the screen level. Figure 4 presents some images of the measurements conducted. Lee et al. [56] used Electrical resistance tomography (ERT) to evaluate the uniformity of liquor flow through a porous media in a laboratory model digester under scaled industrial conditions: a 1:15 geometrically scaled vessel, a vessel to particle diameter ratio of 93:1 to minimise wall effects, and close approximation of liquor superficial velocity and pore Reynolds number (pore Reynolds number is based on the capillary model of flow through packed beds and accounts for the porosity, $\varepsilon$, tortuosity, $\tau$, and specific surface area presented to the flow). Their motivation departed from the literature, where the extent of delignification in batch digesters varied as a function of chip location in the vessel. This non-uniformity may be exacerbated by several factors but is commonly attributed to poor and/or non-uniform liquor flow through the reactor. Local interstitial flow velocities were measured using pixel-pixel cross correlation techniques. Tests were conducted to cover the range of typical liquor splits, $Q_{T} / Q_{B}$ (the liquor volumetric flow ratio between the top and bottom of the digester) found in industrial units: $0.5,1.0$, and 2.0. The results showed that creation of uniform zones was readily achieved, although a certain flow rate was needed in the upper section to establish uniformity due to asymmetric liquor addition to the vessel. A mixing region was observed at the screen elevation in the outflow zone from the digester. The location of this interaction zone moved, depending on the relative liquor flux to the top and bottom of the vessel. When local flow velocities were measured using the tomographic data (equal liquor fluxes to the top and bottom circulation zones) no flow crossed the screen level. Thus, a stagnation point existed at the centre of the vessel near the plane of the extraction screens. However, by forcing liquor to pass through the screen plane (by directing the flow from the top to the bottom of the digester and bypassing the circulation screens, or vice versa) during part of the cook, the spatial dependence of kappa number (related to the degree of cooking) should be reduced.

Hui and co-workers [127] studied the effect of non-Newtonian rheology, including the presence of yield stress, characteristic of pulp fibre in mixing operations, where regions of active motion around the impellers can be created with the cavern size affecting the quality of mixing attained. A scaled version of a commercial axial flow impeller was used in a standard side-entering configuration. Measured cavern diameters were compared against model predictions available in the literature. Since the studied pulp suspensions were opaque, cavern size had to be imaged using indirect techniques for which two methods were used, being ERT one of them (the other was ultrasonic Doppler velocimetry, UDV): ERT was the preferred one due to the marked decrease in the time required to acquire data. The shape of the cavern was best approximated as a truncated right-circular cylinder and, as expected, increasing the impeller speed increased the cavern volume. However, the development of the cavern volume with increasing impeller speed was not uniform, which was attributed to the interaction between the cavern and the vessel walls. A model was developed for predicting cavern development in yield stress fluids which included interaction with vessel boundaries. The proposed model predicts well the trend 
in cavern volume change with increasing impeller speed although the absolute cavern volume is only predicted to within $13 \%$.

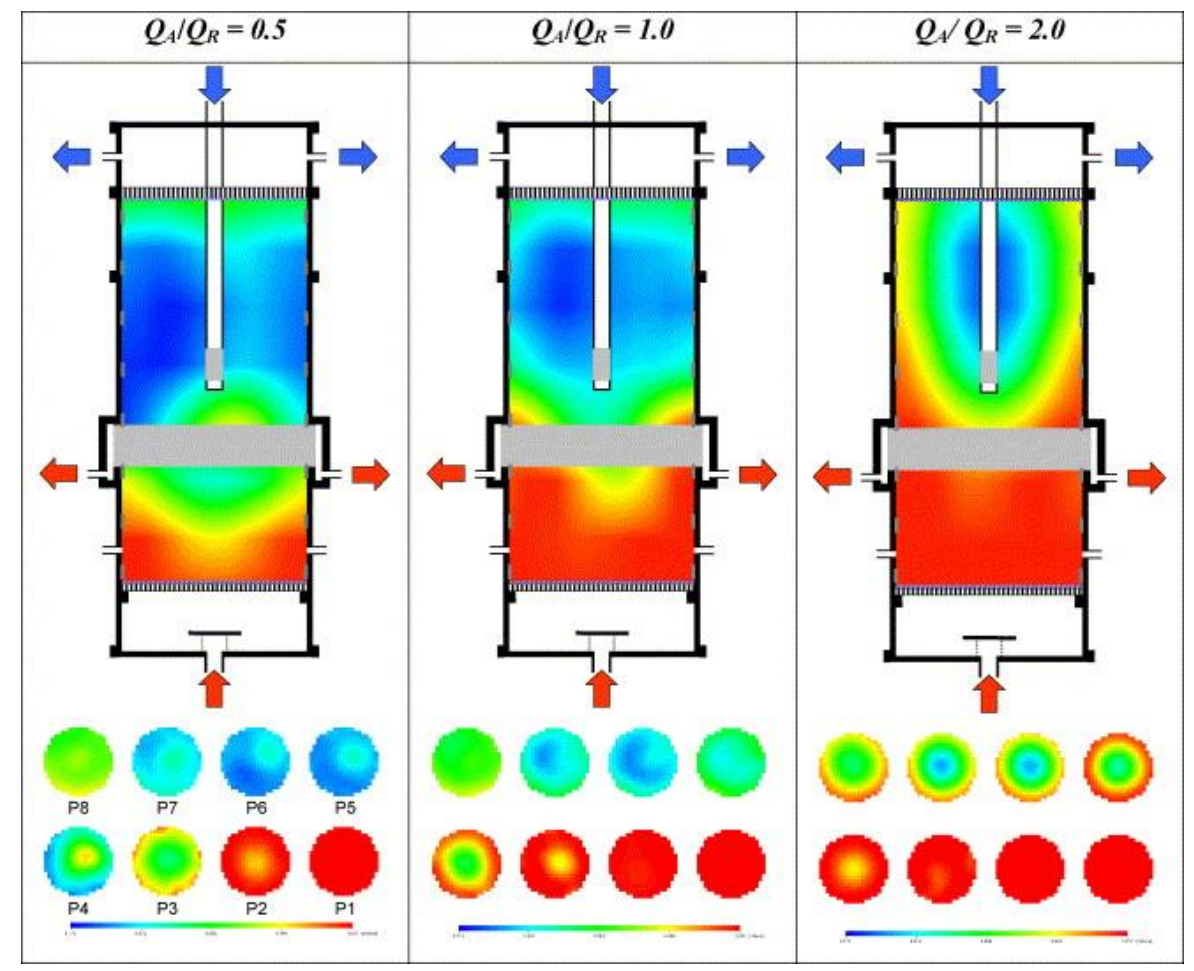

Figure 4. Tomographic images of a liquid with a certain concentration, a liquor, flowing through a packed bed of HDPE pellets at steady flow conditions, obtained using a set of sensing electrode rings located sequentially along a pipe (the planes are numbered from the bottom, starting with number 1). A step reduction in liquid conductivity was made in the flow exiting the down comer. Images display the liquid concentration distribution at steady-state flow ratios of $Q_{A} / Q_{R}$ of $0.5,1.0$ and 2.0. A total liquid flux of $75 \mathrm{~mL} / \mathrm{s}$ was used for $Q_{A} / Q_{R}$ of 0.5 and 2.0 , and of $50 \mathrm{~mL} / \mathrm{s}$ for $Q_{A} / Q_{R}$ equal to 1.0. In the tomographic diagrams, low conductivity regions are coloured blue and high conductivity regions are coloured red (Reproduced with permission from Ruzinsky et al. [126]).

\subsection{Pulp Suspensions Characterisation Using ET: The Authors' Approach}

\subsubsection{Overview of the Developed EIT System and of the Experimental Setup}

The authors of this paper have also been working on the improvement of a proprietary EIT system used in data acquisition of two-phase flows (solid/liquid). The reconstructed images aim to validate the CFD developed model $[74,112,128]$, particularly when applied to the flow characterisation of pulp suspensions. To obtain improved sensitivity, the developed EIT system determines the differences in both the real and imaginary parts of the impedance over the domain. It is a portable device; consequently, it can be used in any location where 16 or 32 electrode planes have been installed on pipes. To avoid the cost and difficulties inherent in the construction of a current source, a voltage source was projected instead, a choice different from what is typical when designing electrical tomography apparatus. Jia et al. [67], demonstrated that the use of a potential voltage difference instead of current in the domain excitation procedure made it possible to surpass the limitations found when studying more conductive media. The developed EIT system has already been described previously [74]; nevertheless, together with a block diagram of the system architecture and its main modules, presented in Figure 5, a brief description is given here. A set of input/output boards with USB ports (DAQ boards) are used for interfacing the EIT system with a personal computer (PC). Using the analogue input channels of the DAQ boards, the electrical potentials, resulting from each applied stimulus, are read. The developed 
EIT system can acquire data at a rate that allows an image reconstruction rate of up to 4000 or 1000 frames per second, depending on the number of electrodes composing the sensing rings, either 16 or 32. To obtain the desired excitation signals, with the desired output amplitude and frequency (up to $25 \mathrm{MHz}$ for this system), a programmable frequency voltage waveform was used. The authors also adopted an excitation strategy based on a voltage signal instead of a current, as has already been mentioned, as the use of this approach resulted in less noisy data [73]. In parallel with the generation of the stimulus signal, two others were also created, both with unitary magnitude, but where one is in phase with the excitation signal and the other is 90 degrees out of phase. To demodulate the read electrical potentials, each of them is multiplied simultaneously by both in phase and out of phase unitary produced signals; the signals outputted by the multipliers go through low pass filters, enabling only the constant terms to be extracted from them, which are the terms needed to calculate the impedance distribution in the domains. A set of analogue bidirectional multiplexers are used for routing of all signals: the excitation is directed to the desired electrode pair, and the read electric potentials to the multipliers. The digital outputs of the DAQ boards are used to address the multiplexers. This EIT system was used to characterise pulp suspensions in flow tests conducted in a pilot rig described elsewhere [129] and schematically shown in Figure 6. In short, the test section consisted of a horizontal PVC pipe, $7.5 \mathrm{~m}$ in length and $100 \mathrm{~mm}$ internal diameter.

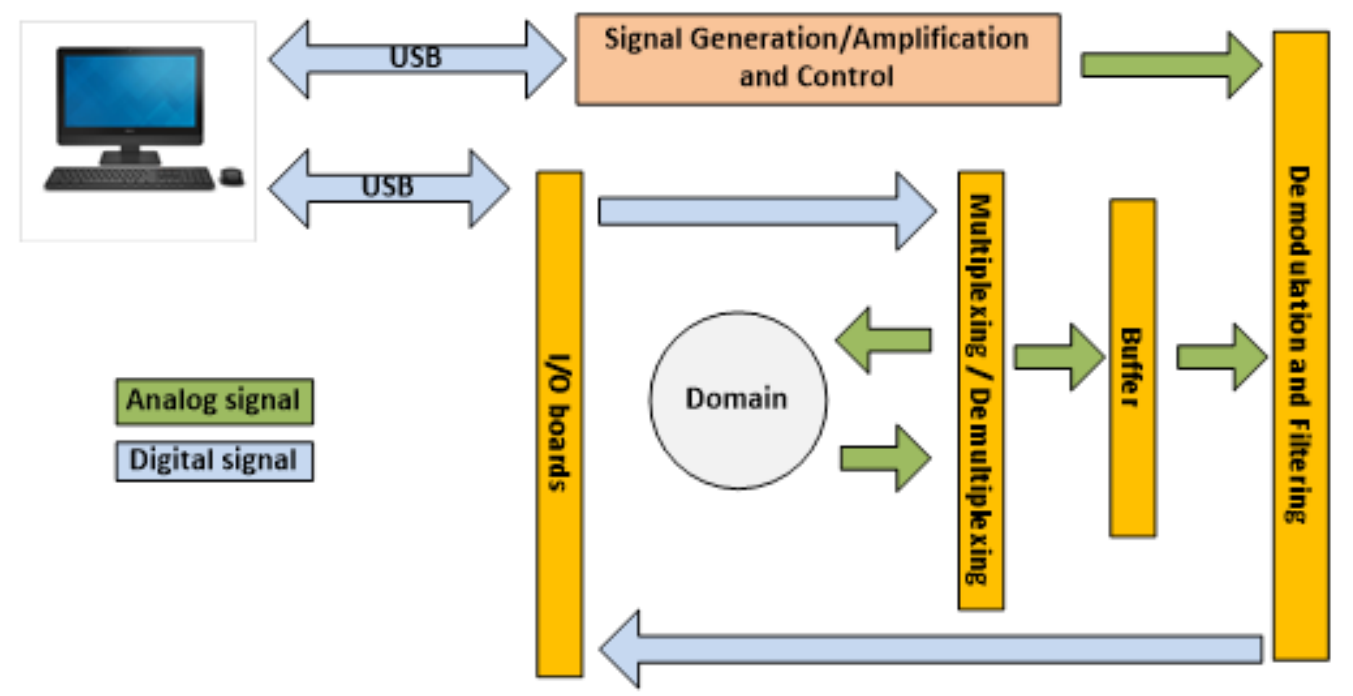

Figure 5. Block diagram of the actual tomographic system main modules.

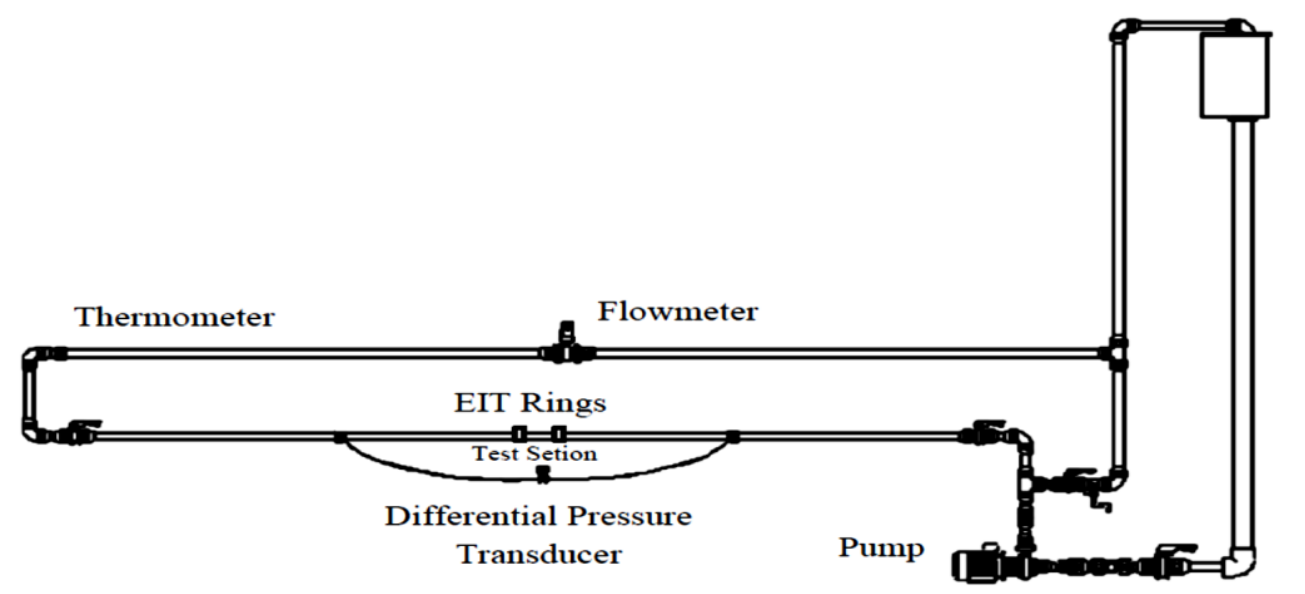

Figure 6. Pilot rig schematic.

The flow rate could be regulated through the manipulation of two valves located after the pump and was measured by an electromagnetic flow meter. Appropriate lengths were inserted before and 
after the test section to account for entrance and exit effects. The EIT electrodes rings (constructed in Teflon), see Figure 7, consisted of 32 Titanium-based electrodes (each with a diameter of $5 \mathrm{~mm}$ which was previously optimised experimentally), circumferentially and equally spaced, and mounted in a Perspex tube inserted in the rig between the measuring pressure taps. For all tests reported here, an excitation frequency of $10 \mathrm{kHz}$ with $2 \mathrm{~V}_{\mathrm{pp}}$ amplitude was imposed, and opposite injection and adjacent measuring protocols were used. For image reconstruction, the open-source software EIDORS [130], considering direct differential measured voltages and using a structured Mesh consisting of 2304 linear elements and 1201 nodes, was used. EIDORS implements a non-linear back projection method using a regularised algorithm (Tikhonov regularisation). To solve the forward problem the Complete Electrode model (CEM) was chosen [131]. In the reconstructed images of the conductivity distribution in the pipe cross-section, electrode number 1 corresponds to the top position in the cross-section of the tube; a darker blue colour corresponds to a lower conductivity region, where the concentration of the fibres is higher, while yellow or red colours identify higher conductivities regions, where the fibres are present in lower concentrations.

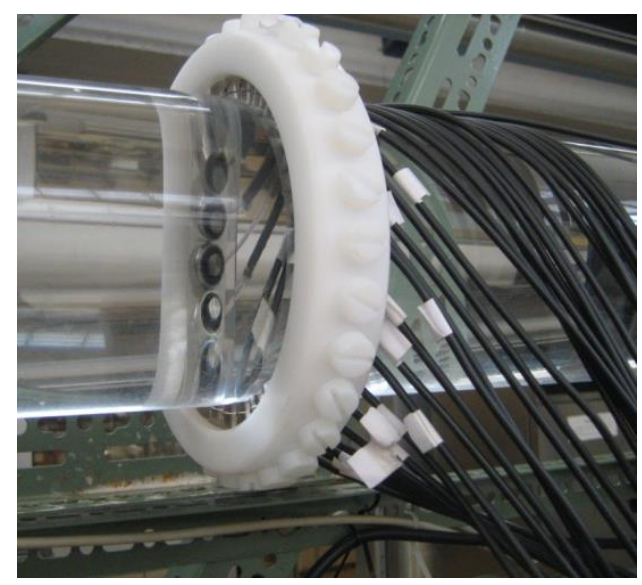

Figure 7. Image of one of the 32 Titanium electrodes test rings.

\subsubsection{Overview of the Pulp Suspension Flow Imaging}

For the tests depicted in this section, industrial pulp suspensions of Pine and Eucalyptus fibres, both collected in a local paper mill, were firstly squeezed and washed in water, and the fibres were then resuspended in tap water doped with $\mathrm{NaCl}$ with adjusted electrical conductivity.

The behaviours of Eucalyptus pulp suspensions (average Fibre length of $0.706 \mathrm{~mm}$ ) and of Pine pulp suspensions (average Fibre length of $2.18 \mathrm{~mm}$ ) were compared, as in Figure 8, under the conditions listed in Table 1.

Table 1. Table of concentrations and flow velocities tested.

\begin{tabular}{cccc}
\hline Type of Pulp & $\begin{array}{c}\text { Concentration } \\
(\mathbf{w t} / \mathbf{w t})\end{array}$ & Flow Velocities $(\mathbf{m} / \mathbf{s})$ & $\begin{array}{c}\text { Suspension } \\
\text { Conductivity }\left(\mathbf{m s} \cdot \mathbf{c m}^{-\mathbf{1}}\right)\end{array}$ \\
\hline Eucalypt & 2.35 & $0.5,1,1.5,2,2.5,3$ & 1.460 \\
Pine & 1.5 & $0.5,1,1.5,2,2.5,3$ & 1.150 \\
& 1 & $0.5,1,1.5,2,2.5,3$ & 1.128 \\
& 2.35 & $0.5,1,1.5,2,2.5,3$ & 1.471 \\
& 1.5 & $0.5,1,1.5,2,2.5,3$ & 1.461 \\
1 & $0.5,1,1.5,2,2.5,3$ & 1.435 \\
\hline
\end{tabular}

Before analysing the obtained suspensions flow images, we must reinforce that the tomography's reflect the voltage differences measured. For fibre concentrations of around $2.35 \%$, the fibre distributions in the cross-pipe section are diverse for the two types of fibres. Concerning the Pine suspension, a plug type flow behaviour is already visible at the lowest velocity, with an increase in the plug (size and 
intensity of the blue colour) when the velocity increases to $2 \mathrm{~m} / \mathrm{s}$ (see Figure $8 \mathrm{a}, \mathrm{c}, \mathrm{e}$ ). However, a further increase in the velocity up to $3 \mathrm{~m} / \mathrm{s}$ leads to breakage of the plug, as in Figure 8g), as was expected for this type of long and stiff fibre.

a)

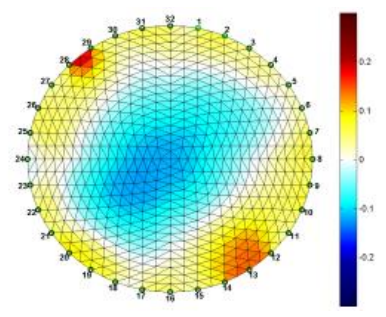

c)

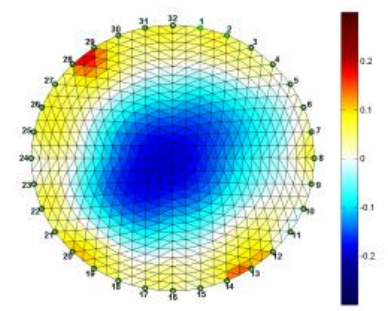

e)

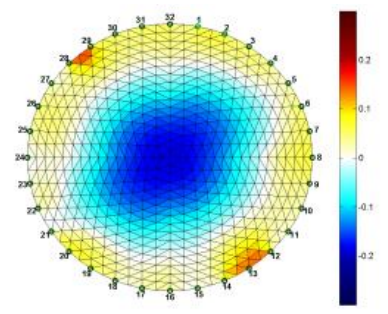

g)

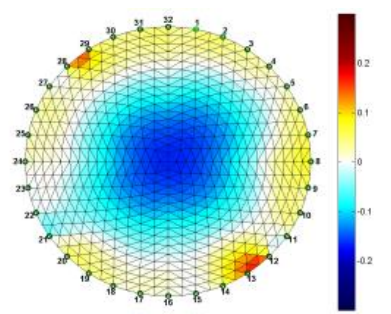

b)

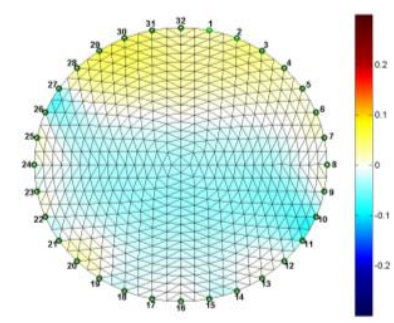

d)

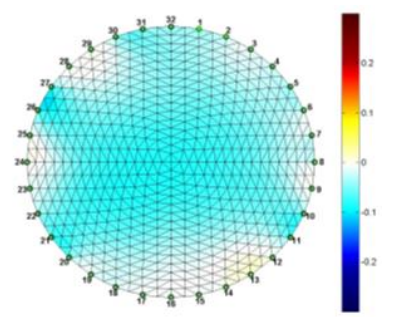

f)

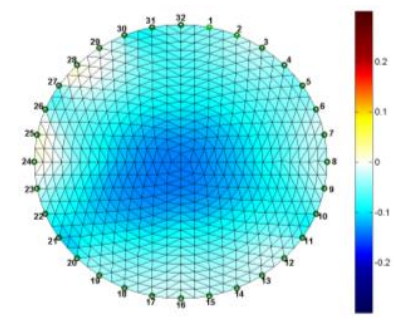

h)

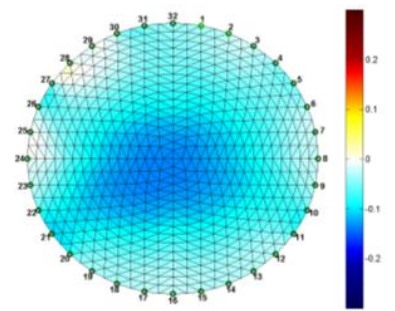

Figure 8. EIT images obtained with a concentration of $\cong 2.35 \%(w / w)$ of Pine (left column: $(\mathbf{a}, \mathbf{c}, \mathbf{e}, \mathbf{g})$ ) and Eucalyptus (right column: (b,d,f,h)) fibres with increasing flow velocity $(0.5 \mathrm{~m} / \mathrm{s}, 1 \mathrm{~m} / \mathrm{s}, 2 \mathrm{~m} / \mathrm{s}$ and $3 \mathrm{~m} / \mathrm{s}$, top to bottom).

When analysing the Eucalyptus fibre suspension behaviour, at the lowest velocity the fibres remain more or less dispersed in the cross-pipe section (see Figure $8 b$ ), and the plug only starts to be visible at the velocity of $1 \mathrm{~m} / \mathrm{s}$, becoming denser at $2 \mathrm{~m} / \mathrm{s}$ (Figure $8 \mathrm{~d}, \mathrm{f}$ ).

Similarly to the Pine fibres, a further increase in the Eucalyptus suspension velocity up to $3 \mathrm{~m} / \mathrm{s}$ leads to plug breakage (Figure 8h). These differences in the reconstructed EIT images for this concentration are related to the physical properties of the fibres, in particular the fibre length and stiffness of the two pulps that originate different interactions between fibres when the suspensions flow in the tube.

For a fibre concentration of around 1\% (EIT images depicted in Figure 9), it was not possible to collect consistent data for the Eucalyptus fibres, and so Figure 9 depicts EIT images only for Pine fibre suspensions. For these suspensions, and by comparison with Figure 8 , it is evident that at lower concentrations and lower velocities (in this case $0.5 \mathrm{~m} / \mathrm{s}$ ), Pine fibres migrate and become agglomerated in a central plug, while for a more a more concentrated suspension, the fibres (Figure 8) occupy a larger region of the pipe cross-section. However, at more diluted suspensions, the strength of 
the interconnection of the fibres is lower and the plug breakage occurs at lower velocities as the velocity increases.

a)

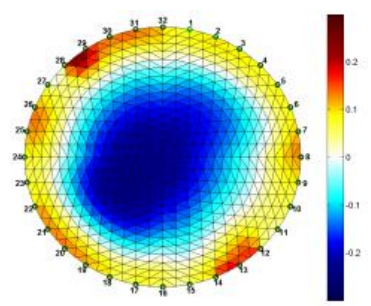

c)

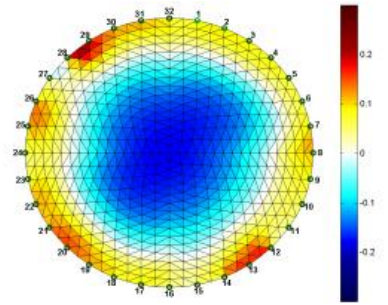

b)

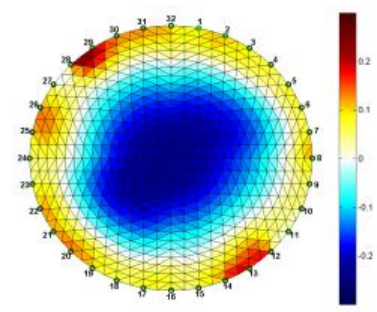

d)

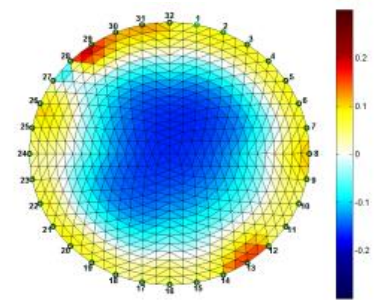

Figure 9. EIT images obtained with a concentration of $\cong 1 \%(w / w)$ of Pine fibres (a-d) for $0.5 \mathrm{~m} / \mathrm{s}, 1 \mathrm{~m} / \mathrm{s}$, $1.5 \mathrm{~m} / \mathrm{s}$ and $2.5 \mathrm{~m} / \mathrm{s}$, respectively.

It must be emphasised at this point that environmental factors that were not easily kept under control may affect the colour mapping of the reconstructed images. In particular, the temperature changes significantly during continuous testing due to the energy input from the pump. Moreover, since cooling of the system was performed by passing tap water through a tube immersed in the suspension tank, the temperature of this cooling water changes significantly with respect to the seasons, which also affects the temperature in the rig. Changes in the suspension temperature affect the viscosity, but mainly the electric conductivity of the suspension and, consequently, the colour map of the reconstructed images is affected.

\subsubsection{Fibre Flow Velocity Estimation}

By using two EIT planes and cross-correlation, EIT can also be used to determine the velocity profile in a pipe. For example, Mosorov et al. [132] used a best-correlated pixel method to measure both axial and radial velocities simultaneously. Independently of the procedure used, a marker is needed. As a first approach, the introduction of a small fraction of conductive or non-conductive solid spheres in the flow was used. After several attempts without success to distinguish these markers in the EIT images, the authors instead decided to use a trace of a highly concentrated $\mathrm{NaCl}$ solution as a marker. For the purpose of introducing this $\mathrm{NaCl}$ solution in the flowing fibre suspension, an injection system was developed and added to the existing pilot rig: it was located around $3 \mathrm{~m}$ before the first EIT electrode ring. This injector was built in such a way that the flow suffered almost no disturbance. For all the tests reported in this section, two 16 electrode titanium rings A and B were used, $30 \mathrm{~cm}$ apart, with ring A closer to the injector inlet, as shown in Figure 10. The procedure here reported for estimating velocity was different from those reported in the literature, but, nevertheless, with some similarities with the one described by Dong et al. [133]. Instead of using the complete set of EIT projections, the authors only made use of the first projection; first, the potential differences of each pair of the first projection with no marker injection in the flow were acquired, afterwards, they were subtracted to the measured voltage differences obtained during the runs with $\mathrm{NaCl}$ injection.

Finally, the sum of the module of those differences was computed. For each suspension velocity and fibre concentration tested, several injection trials were performed to assess the reproducibility of this approach. The conditions used for the velocity estimation are summarised in Table 2. Some examples of data obtained with this approach are shown in Figure 11: three sets of $\mathrm{NaCl}$ injections for suspensions containing $1 \%$ and $2 \%$ of Eucalyptus fibres with a velocity of $0.25 \mathrm{~m} / \mathrm{s}$ (Figure $11 \mathrm{a}, \mathrm{b}$ ). 


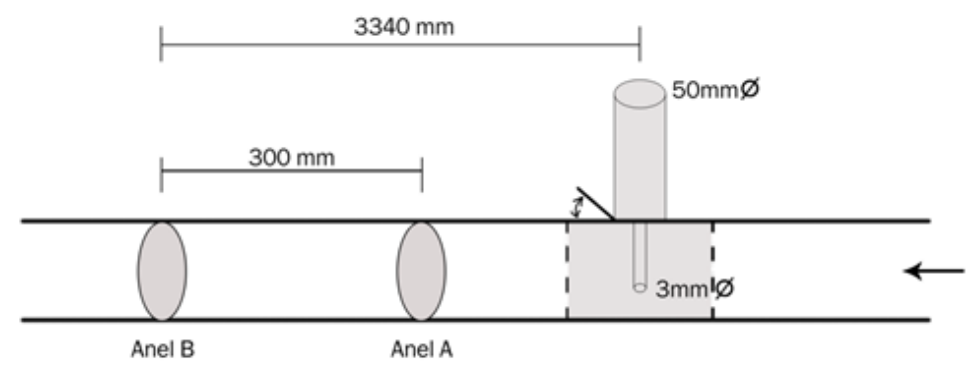

Figure 10. Schematic of the relative position of the EIT electrodes sections and $\mathrm{NaCl}$ injection inlet.

Table 2. Table of fibre concentrations and flow velocities used for the velocity estimation tests.

\begin{tabular}{cc}
\hline Concentration $(\mathbf{w t} / \mathbf{w t})$ & Flow Velocities $(\mathrm{m} / \mathbf{s})$ \\
\hline 3 & $0.25,0.5$ \\
2 & $0.25,0.5,0.75,1$ \\
1 & $0.25,0.5,0.75,1$ \\
\hline
\end{tabular}

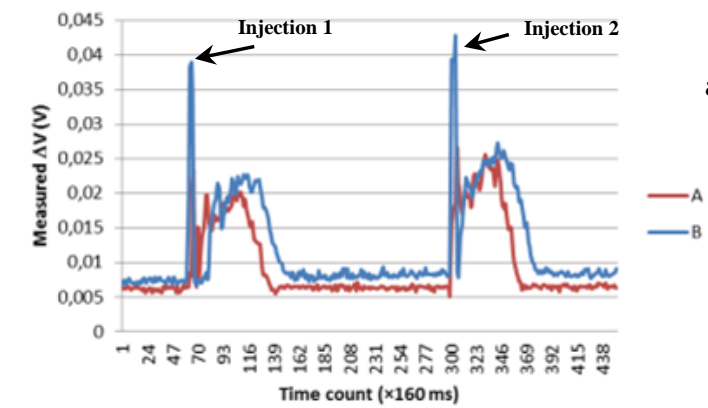

a)
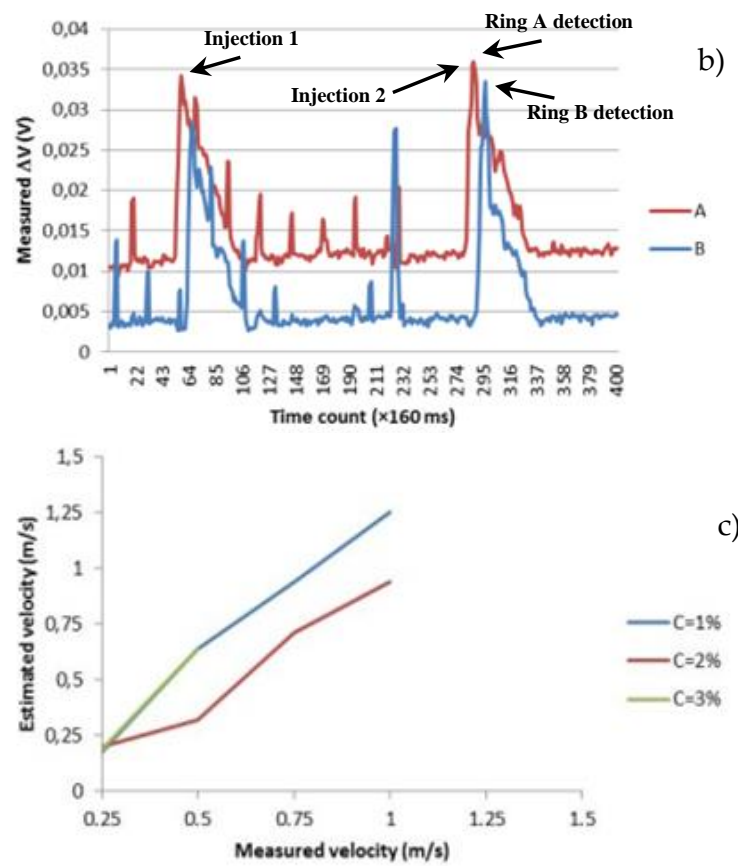

Figure 11. EIT injection test trials for fibre suspension concentrations of $1 \%$ and $2 \%$, at the velocity of $0.25 \mathrm{~m} / \mathrm{s}(\mathbf{a}, \mathbf{b})$, and estimated velocity versus measured velocity for all fibre concentrations (c).

In that same figure, a graph showing the estimated velocity versus the measured suspension velocity is represented (Figure 11c). A quite reasonable estimation is obtained, and so the authors' approach was validated. Moreover, as fibre concentration increases, the advance of the $\mathrm{NaCl}$ front is slowed due to the presence of more fibres and to the structures that build up among fibres. This can 
be observed between Figure 11a,b, where for the higher fibre concentration, there is a delay in the detection of the $\mathrm{NaCl}$ in the second ring (ring B). Therefore, in the future, it can be used to evaluate those structures as a function of fibre concentration and flow velocity.

\section{Conclusions}

Theoretical models developed to describe multiphase flows have been developed that have different assumptions requiring experimental verification for validation. For example, reliable data on velocity profiles in concentrated pulp suspensions during pipe flow is scarce in the literature. A multitude of techniques is now available, but a condition of non-disturbance of the flows is required in view of the complexity of multiphase systems. Non-invasiveness is therefore beneficial and is characteristic of some of these techniques. Laser Doppler Anemometry (LDA), Magnetic Resonance Imaging (MRI), Ultrasonic Velocity Profiling (UVP) and Electrical Tomography were reviewed and compared for their energy requirements, spatial and temporal resolution, duration of observation times, spatial and temporal coherence and stability, cost of equipment, and field and scale of applications. The compromise between the advantages and limitations of these techniques led us to focus on the Electric Tomographic techniques, the principles behind them, and their application in the visualisation of pulp suspension flows. Our efforts were directed towards designing proprietary, low-cost, portable Electric Impedance Tomographic (EIT) hardware. To circumvent the difficulties and costs of designing a current source that suits the goals of the EIT system being designed, the choice was made to move from traditional EIT systems and, instead, to design a voltage source. The EIT system depicted here can be used with 16-electrode or 32-electrode rings. Examples of Eucalyptus and Pine pulp suspension flows are reported here, demonstrating the influence of fibre concentration and type, as well as flow velocity, on the distribution of the fibres in the cross-sectional area of a horizontal pipe.

The developed Electrical Impedance Tomography system was used to characterise the behaviour of pine and eucalyptus fibre suspension flow through a pipe at different fibre concentrations- $1 \%, 1.5 \%$ and $2.35 \%(\mathrm{w} / \mathrm{w})$ - and at varying flow velocities (between 0.5 and $3 \mathrm{~m} / \mathrm{s}$ ) based on the cross-section images obtained for totally developed flows. Especially for the pine fibre suspensions, it was clearly possible to identify a plug formation, which was probably due to the geometry of the pine fibres, which were longer than the eucalyptus ones. With respect to their usage for the estimation of suspension velocity, eucalyptus fibre suspensions were used in three different concentrations- $1 \%, 2 \%$ and $3 \%$ $(\mathrm{w} / \mathrm{w})$ - and at velocities of between 0.25 and $1 \mathrm{~m} / \mathrm{s}$. The real suspension velocity measured by a flowmeter was quite reasonably estimated by the cross correlation of EIT images obtained in two consecutive electrode rings separated from one another by $300 \mathrm{~mm}$.

The developed EIT system allows 2D image reconstruction rates of up to 4000 or 1000 frames per second, depending on the number of electrodes used in the acquisition (16 or 32). With respect to the resolution of the obtained cross-section images, it is much higher than previously reported in the literature (which is evident by comparing Figure 3 [53] and Figure 8).

Thus far, the authors have used the developed equipment not only in the monitoring of fibre suspension flow and the estimation of fibre flow velocity, but also with great success in other diverse two-phase systems (solid-liquid [72], liquid-liquid [111], just to mention two). In the next step, the objective will be to upgrade the overall system (signal acquisition and image reconstruction algorithms) to visualise three-phase systems.

Considering the results presented here, the usefulness of this technique and equipment is demonstrated and clearly justifies its use at an industrial scale, despite its lower spatial resolution when compared with other tomographic procedures.

Author Contributions: All authors contributed equally to this work. All authors have read and agreed to the published version of the manuscript.

Funding: This research was funded by COST Action FP1005, Fibre suspension flow modelling-A key for innovation and competitiveness in the pulp \& paper industry, whose support is gratefully acknowledged. The authors thank the financial support of Fundação para a Ciência e Tecnologia through the project, contract 
PTDC/EQU-EQU/112388/2009 (COMPETE-01-0124-FEDER-015247), and contracts Pest-C/EME/UI0285/2013 and Pest-C/EQB/UI0102/2013, both financed by FCT/MCTES (PIDDAC) and co-financed by the European Regional Development Fund through the program COMPETE.

Conflicts of Interest: The authors declare no conflict of interest.

\section{References}

1. Steiner, G.; Podd, F. A non-invasive and non-intrusive ultrasonic transducer array for process tomography. In Proceedings of the XVIII IMEKO WORLD Congress on Metrolofy and Sustainable Development, Rio de Janeiro, Brazil, 17-20 September 2006; pp. 17-22.

2. Ijaz, U.Z.; Kim, J.-H.; Khambampati, A.K.; Kim, M.-C.; Kim, S.; Kim, K.-Y. Concentration distribution estimation of fluid through electrical impedance tomography based on interacting multiple model scheme. Flow Meas. Instrum. 2007, 18, 47-56. [CrossRef]

3. Murphy, S.C.; York, T.A. Electrical impedance tomography with non-stationary electrodes. Meas. Sci. Technol. 2006, 17, 3042-3052. [CrossRef]

4. Durst, F.; Melling, A.; Whitelaw, J.H. Principles and Practice of Laser-Doppler Anemometry; Academic Press: New York, NY, USA, 1981.

5. Chaouki, J.; Larachi, F.; Dudukovic, M.P. Non-invasive tomographic and velocimetric monitoring of multiphase flows. Ind. Eng. Chem. Res. 1997, 36, 4476-4503. [CrossRef]

6. Takeda, Y. Velocity Profile Measurement by Ultrasonic Doppler Shift Method. In Proceedings of the National Heat Transfer Conference of the American Society of Mechanical Engineers, Philadelphia, PA, USA, 6-9 August 1989; pp. 155-160.

7. Haavisto, S.; Cardona, M.; Salmela, J.; Powell, R.; McCarthy, M.; Kataja, M.; Koponen, A. Experimental investigation of the flow properties and rheology of complex fluids in pipe flows by hybrid multiscale velocimetry. Exp. Fluids 2017, 58, 158. [CrossRef]

8. Sorokin, M.; Strokina, N.; Eerola, T.; Lensu, L.; Karttunen, K.; Kalviainen, H. Image-based characterisation of the pulp flows. Pattern Recognit. Image Anal. 2016, 26, 630-637. [CrossRef]

9. Heindel, T. A review of X-ray flow visualisation with applications to multiphase flows. J. Fluids Eng. 2011, 133. [CrossRef]

10. Schlieper, G. Principles of Gamma ray densitometry. Met. Powder Rep. 2000, 55, 20-23. [CrossRef]

11. Yenjaichon, W.; Grace, J.R.; Lim, C.J.; Bennington, C.P.J. Characterisation of gas mixing in water and pulp-suspension flow based on electrical resistance tomography. Chem. Eng. J. 2013, 214, 285-297. [CrossRef]

12. Nakagawa, M.; Altobelli, S.A.; Caprihan, A.; Fukushima, E.; Jeong, E.K. Non-invasive measurements of granular flows by magnetic resonance imaging. Exp. Fluids 1993, 16, 54-60. [CrossRef]

13. Sederman, A.J.; Gladden, L.F.; Mantle, M.D. Application of magnetic resonance imaging techniques to particulate systems. Adv. Powder Technol. 2007, 18, 23-38. [CrossRef]

14. Müller, C.R.; Holland, D.J.; Sederman, A.J.; Mantle, M.D.; Gladden, L.F.; Davidson, J.F. Magnetic resonance imaging of fluidized beds. Powder Technol. 2008, 183, 53-62. [CrossRef]

15. Li, T.Q.; Ödberg, L. Flow properties of cellulose fiber suspensions flocculated by cationic polyacrylamide. Colloids Surf. A Physiochem. Eng. Asp. 1996, 115, 127-135. [CrossRef]

16. Li, T.Q.; Ödberg, L. Studies of Flocculation in Cellulose Fibre Suspensions by NMR Imaging. J. Pulp Pap. Sci. 1997, 23, 401-405.

17. Li, T.Q.; Powell, R.L.; Ödberg, L.; McCarthy, M.J.; McCarthy, K.L. Velocity measurements of fiber suspensions by the nuclear magnetic resonance imaging method. Tappi J. 1994, 77, 145-149.

18. Li, T.Q.; Seymour, J.D.; Powell, R.L.; McCarthy, M.J.; McCarthy, K.L.; Ödberg, L. Visualization of flow patterns of cellulose fiber suspensions by NMR imaging. Aiche J. 1994, 40, 1408-1411. [CrossRef]

19. Li, T.Q.; Weldon, M.; Ödberg, L.; McCarthy, M.J.; Powell, R.L. Pipe flow behaviour of hardwood pulp suspensions studied by NMRI. J. Pulp Pap. Sci. 1995, 21, 408-414.

20. Arola, D.F.; Li, T.Q.; Ödberg, L.; Powell, R.L.; McCarthy, M.J. Flow of pulp suspensions through a sudden expansion. Aiche J. 1998, 44, 2597-2606. [CrossRef]

21. Seymour, J.D.; Maneval, J.E.; McCarthy, K.L.; McCarthy, M.J.; Powell, R.L. NMR velocity phase encoded measurements of fibrous suspensions. Phys. Fluids A Fluid Dyn. 1993, 5, 3010-3012. [CrossRef] 
22. Slavica, R. Laser Doppler Anemometry and its Application in Wind Tunnel Tests. Sci. Tech. Rev. 2007, LVII, 64-76.

23. Ek, R.; Möller, K.; Norman, B. Measurement of velocity and concentration variations in dilute fiber/air suspensions using a laser Doppler anemometer. Tappi J. 1978, 61, 49-52.

24. Kerekes, R.J.; Garner, R.G. Measurement of Turbulence in Pulp Suspensions by Laser Anemometry. Trans. Tech. Sect. 1982, CPPA 8, TR53.

25. Steen, M. The Application of Refractive Index Matching for Two-phase Flow Measurements in Turbulent Fibre Suspensions by Laser Doppler Anemometry. Nord. Pulp Pap. Res. J. 1989, 4, 236-243. [CrossRef]

26. Steen, M. On Turbulence Structure in Vertical Pipe Flow of Fiber Suspensions. Nord. Pulp Pap. Res. J. 1989, 4, 244-252. [CrossRef]

27. Andersson, S.; Rasmuson, A. Flow Measurements on a Turbulent Fibre Suspension by Laser Doppler Anemometry. Aiche J. 2000, 46, 1106-1119. [CrossRef]

28. Pettersson, J.; Rasmuson, A. LDA Measurements on a Turbulent Gas/Liquid/Fibre Suspension. Can. J. Chem. Eng. 2004, 82, 265-274. [CrossRef]

29. Pettersson, J.; Wikström, T.; Rasmuson, A. Near Wall Studies of Pulp Suspension Flow Using LDA. Can. J. Chem. Eng. 2006, 84, 422-430. [CrossRef]

30. Takeda, Y. Velocity Profile Measurement by Ultrasonic Doppler Method. Exp. Fluid Sci. 1995, 10, 444-453. [CrossRef]

31. Wiklund, J.; Pettersson, J.; Stading, M.; Rasmuson, A. A Comparative Study of UVP and LDA Techniques for Pulp Suspensions in Pipe Flow. Aiche J. 2006, 52, 484-495. [CrossRef]

32. Ozaki, Y.; Kawaguchi, T.; Takeda, Y.; Hishida, K.; Maeda, M. High time resolution ultrasonic velocity profiler. Exp. Fluid Sci. 2002, 26, 253-258. [CrossRef]

33. Hirsimäki, O. Determination of Radial Velocity Profile and Flow Disturbance of Pulp Suspension by Ultrasonic Echo Correlation. Pap. Ja Puu 1978, 60, 95-97.

34. Karema, H.; Kataja, M.; Kellomäki, M.; Salmela, J.; Selenius, P. Transient Fluidisation of Fibre Suspension in Straight Channel Flow. In Proceedings of the TAPPI Internation Paper Physics Conference, San Diego, CA, USA, 26-30 September 1999; pp. 369-379.

35. Karema, H.; Salmela, J.; Tukiainen, M.; Lepomäki, H. Prediction of Paper Formation by Fluidisation and Reflocculation Experiments. In Proceedings of the 12th Fundamental Resesearch Symposium, Oxford, UK, 11 October 2001; pp. 559-589.

36. Wiklund, J.; Johansson, M.; Shaik, J.; Fischer, P.; Windhab, E.; Stading, M.; Hermansson, A.-M. In-Line Ultrasound based Rheometry of industrial and model suspensions flowing through pipes. In Proceedings of the Third International Symposium on Ultrasonic Doppler Methods for Fluid Mechanics and Fluid Engineering, Lausanne, Switzerland, 9-11 September 2002; pp. 69-76.

37. Claesson, J.; Rasmuson, A.; Wiklund, J.; Wikström, T. Measurement and analysis of flow of concentrated fiber suspensions through sudden expansion using UVP. Aiche J. 2013, 59, 1012-1021. [CrossRef]

38. Kotzé, R.; Wiklund, J.; Haldenwang, R. Optimisation of Pulsed Ultrasonic Velocimetry system and transducer technology for industrial applications. Ultrasonics 2013, 53, 459-469. [CrossRef] [PubMed]

39. Xu, H.; Aidun, C. Characteristics of fiber suspension flow in a rectangular channel. Int. J. Multiph. Flow 2005, 31, 318-336. [CrossRef]

40. Dresxler, W.; Fujimoto, J. Optical Coherence Tomography: Technology and Applications; Springer: Berlin, Germany, 2008.

41. Kataja, M.; Haavisto, S.; Slamela, J.; Letho, R.; Koponen, A. Characterisation of micro-fibrilated cellulose fiber suspension flow using multi scale velocity profile measurements. Nord. Pulp Pap. J. 2017, 31, 473-482. [CrossRef]

42. Fujimoto, J.; Pistris, C.; Boppart, S.; Brezinski, M. Optical coherence tomography: An emerging techonology for biomedical imaging and optical biopsy. Neoplasia 2000, 2, 9-25. [CrossRef]

43. Gao, Q.; Wang, H.; Shen, G. Review on development of volumetric particle image velocimetry. Chin. Sci. Bull. 2013, 58, 4541-4556. [CrossRef]

44. Fan, L.; Xu, N. Study on laminar flow containing fibre particles in a stirred tank using DPI. Powder Technol. 2017, 320, 420-427. [CrossRef]

45. Fock, H.; Claesson, J.; Rasmusson, A.; Wikström, T. Near wall effect in the plug flow of pulp suspensions. Can. J. Chem. Eng. 2011, 89, 1207-1216. [CrossRef] 
46. Farrington, T. Soft X-Ray Imaging Can Be Used to Assess Sheet Formation and Quality. Tappi J. 1988, 71 , 140-144.

47. Heindel, T.; Monefeldt, J. Flash X-Ray Radiography for Visualizing Gas Flows in Opaque Liquid/Fiber Suspensions. In Proceedings of the 6th International Symposium on Gas-Liquid Two-Phase Flows, Vancouver, BC, Canada, 15-17 December 1997.

48. Heindel, T.; Monefeldt, J. Observations of the Bubble Dynamics in a Pulp Suspension Using Flash X-Ray Radiography. Tappi J. 1998, 81, 149-158.

49. Akbar, M.K.; Ghiaasiaan, S.M.; Karrila, S. An experimental study of interfacial surface area concentration in a short vertical column subject to paper pulp-water-gas three-phase flow. Chem. Eng. Sci. 2004, 59, 1079-1086. [CrossRef]

50. Babelli, I.M.M. Development of Multiphase Meter Using Gamma Densitometer Concept. In Proceedings of the INC '97-International Nuclear Conference: A new era in Nuclear Science and Technology-The challenge of the 21st century, Kuala Lumpur, Malaysia, 27-28 October 1997.

51. Xie, T.; Ghiaasiaan, S.; Karrila, S.; McDonough, T. Flow regimes and gas holdup in paper pulp-water-gas three-phase slurry flow. Chem. Eng. Sci. 2003, 58, 1417-1430. [CrossRef]

52. Dyakowski, T.; Jeanmeure, L.F.C.; Jaworski, A.J. Applications of electrical tomography for gas-solids and liquid-solids flows: A review. Powder Technol. 2000, 112, 174-192. [CrossRef]

53. Xie, C.G.; Reinecke, N.; Beck, M.S.; Mewes, D.; Williams, R.A. Electrical Tomography Techniques for Process Engineering Applications. Chem. Eng. J. 1995, 56, 127-133. [CrossRef]

54. Yenjaichon, W.; Grace, J.R.; Lim, C.J.; Bennington, C.P.J. Pilot-Scale Examination of Mixing Liquid into Pulp Fiber Suspensions in the Presence of an In-Line Mechanical Mixer. Ind. Eng. Chem. Res. 2013, 52, 485-498. [CrossRef]

55. Vlaev, D.S.; Bennington, C.P.J. Flow uniformity in a model digester measured with electrical resistance tomography. Can. J. Chem. Eng. 2005, 83, 42-47. [CrossRef]

56. Lee, Q.F.; Bennington, C.P.J. Measuring flow velocity and uniformity in a model batch digester using electrical resistance tomography. Can. J. Chem. Eng. 2007, 85, 55-64. [CrossRef]

57. Wilkinson, A.J.; Randall, E.W.; Long, T.M.; Collins, A. The design of an ERT system for 3D data acquisition and a quantitative evaluation of its performance. Meas. Sci. Technol. 2006, 17, 2088-2096. [CrossRef]

58. Maxwell, J.C. A Treatise on Electricity and Magnetism; Clarendon Press: Oxford, UK, 1873; Volume I.

59. Morse, T.D.; Ballou, C.O. The uniformity of fluidisation, its measurement and use. Chem. Eng. Prog. 1951, 47, 199-211.

60. Geldart, D.; Kelsey, J.R. The use of capacitance probes in gas fluidised beds. Powder Tech. 1972, 6, 45-50. [CrossRef]

61. Abouelwafa, M.S.A.; Kendall, E.J.M. Analysis and design of helical capacitance sensors for volume fraction determination. Rev. Sci. Instrum. 1979, 50, 872-878. [CrossRef] [PubMed]

62. Hansen, L.S.; Pedersen, S.; Durdevic, P. Multi-phase flow metering in offshore oil and gas transportation pipelines: Trends and perspectives. Sensors 2019, 19, 2184. [CrossRef] [PubMed]

63. Dyakowski, T.; Johansen, G.A.; Hjertaker, B.T.; Sankowski, D.; Mosorov, V.; Wlodarczyk, J. A Dual Modality Tomography System for Imaging Gas/Solids Flows. Part. Part. Syst. Charact. 2006, 23, 260-265. [CrossRef]

64. Yang, W. Tomographic Imaging based on Capacitance Measurement and Industrial Applications. In Proceedings of the IEEE International Workshop on Imaging on Systems and Techniques, Krakow, Poland, 16-18 October 2007.

65. Azzopardi, B.J.; Abdulkareem, L.A.; Zhao, D.; Thiele, S.; da Silva, M.J.; Beyer, M.; Hunt, A. Comparison between Electrical Capacitance Tomography and Wire Mesh sensor for air/silicone oil flow in a vertical pipe. Ind. Eng. Chem. Res. 2010, 49, 805-8811. [CrossRef]

66. Grootveld, C.J. Measuring \& Modelling of Concentrated Settling Suspensions Using Electrical Impedance Tomography. Ph.D. Thesis, University of Delft, Delft, The Netherlands, 1996.

67. Jia, J.; Wang, M.; Schlaberg, H.I.; Li, H. A Novel Tomographic Sensing System for High Electrically Conductive Multiphase Flow Measurement. Flow Meas. Instrum. 2010, 21, 184-190. [CrossRef]

68. Henderson, R.P.; Webster, J.G. An Impedance Camera for Spatially Specific Measurements of the Thorax. IEEE Trans. Biomed. Eng. 1978, 25, 250-254. [CrossRef]

69. Barber, D.C.; Brown, B.H. Applied Potential Tomography. J. Phys. E Sci. Instrum. 1984, 17, 723-733. [CrossRef] 
70. Wang, M.; Jones, T.F.; Williams, R.A. Visualization of Asymmetric Solids Distribution in Horizontal Swirling Flows Using Electrical Resistance Tomography. Chem. Eng. Res. Des. 2003, 81, 854-861. [CrossRef]

71. Malmivuo, J.; Plonsey, R. Bioelectromagnetism: Principles and Applications of Bioelectric and Biomagnetic Fields; Oxford University Press: New York, NY, USA, 1995.

72. Pakzad, L.; Ein-Mozaffari, F.; Chan, P. Using Electrical Resistance Tomography and Computational Fluid Dynamics Modelling to Study the Formation of Cavern in the Mixing of Pseudoplastic Fluids Possessing Yield Stress. Chem. Eng. Sci. 2008, 63, 2508-2522. [CrossRef]

73. Wang, M. Impedance Mapping of Particulate Multiphase Flows. Flow Meas. Instrum. 2005, 16, $183-189$. [CrossRef]

74. Faia, P.M.; Ferreira, A.R.; Santos, M.J.; Santos, J.B.; Silva, A.P.R.; Rasteiro, M.G.; Garcia, F.A.P. Imaging particulate two-phase flow in liquid suspensions with Electric Impedance Tomography. Part. Sci. Technol. 2012, 30, 329-342. [CrossRef]

75. Karapantsios, T.D.; Papara, M. On the design of electrical conductance probes for foam drainage applications. Assessment of ring electrodes performance and bubble size effects on measurements. Colloids Surf. A Phys. Eng. Asp. 2008, 323, 139-148. [CrossRef]

76. Wang, M.; Mann, R.; Dickin, F.J. Electrical resistance tomography sensing systems for industrial applications. Chem. Eng. Commun. 1999, 175, 49-70. [CrossRef]

77. Lucas, G.P.; Cory, J.; Waterfall, R.C.; Loh, W.W.; Dickin, F.J. Measurement of the solids volume fraction and velocity distributions in solids-liquids flows using dual-plane electrical resistance tomography. Flow Meas. Instrum. 1999, 10, 249-258. [CrossRef]

78. Wand, M.; Yin, W. Measurements of concentration and velocity distribution in miscible liquid mixing using electrical resistance tomography. Trans. Chem. Eng. Res. Des. 2001, 79, 883-886. [CrossRef]

79. Molinari, M. High Fidelity Imaging in Electrical Impedance Tomography. Ph.D. Thesis, University of Southampton, Southampton, UK, 2003.

80. Harikumar, R.; Prabu, R.; Raghavan, S. Electrical Impedance Tomography (EIT) and Its Medical Applications: A Review. Int. J. Soft Comput. Eng. 2013, 3, 193-198.

81. Gisser, D.G.; Isaacson, D.; Newell, J.C. Current Topics in Impedance Imaging. Clin. Phys. Physiol. Meas. 1987, 8, 39-46. [CrossRef]

82. Kotre, C.J. EIT image reconstruction using sensitivity weighted filtered back projection. Phys. Meas. 1994, 15, 125-136. [CrossRef]

83. Yang, W.Q.; Gamio, J.C.; Beck, M.S. A fast iterative image reconstruction algorithm for capacitance tomography. In Proceedings of the Sensors and their Applications VIII, Glasgow, UK, 7-10 September 1997; pp. 47-52.

84. Lionheart, W.R.B. Reconstruction algorithms for permittivity and conductivity imaging. In Proceedings of the Second World Congress on Industrial Process Tomography, Hannover, Germany, 29-31 August 2001; pp. 4-11.

85. Yorkey, T.J.; Webster, J.G.; Tompkins, W. Comparing reconstruction algorithms for electrical-impedance tomography. IEEE J. Biomed. Eng. 1987, 34, 843-852. [CrossRef]

86. Parvareh, A.; Rahimi, M.; Alizadehdakhel, A.; Alsaifari, A.A. CFD and ERT investigations on two-phase flow regimes in vertical and horizontal tubes. Int. Comm. Heat Mass Tranfer 2010, 37, 304-311. [CrossRef]

87. Molinari, M.; Cox, S.J.; Blott, B.H.; Daniell, G.J. Efficient non-linear 3D electrical tomography reconstruction. In Proceedings of the Second World Congress on Industrial Process Tomography, Hannover, Germany, 29-31 August 2001; pp. 424-432.

88. Cho, K.H.; Kim, S.; Lee, Y.J. A fast reconstruction method for the two phase flow visualization. Int. Comm. Heat Mass Transf. 1999, 26, 637-646. [CrossRef]

89. Tapp, H.S.; Wilson, R.H. Developments in low-cost electrical imaging techniques. Process. Contr. Qual. 1997, 9, 7-16.

90. Sun, T.D.; Mudde, R.; Schouten, J.C.; Scarlett, B.; Bleek, C.M. Image reconstruction of an electrical capacitance tomography system using an artificial neural network. In Proceedings of the First World Congress on Industrial Process Tomography, Buxton, UK, 14-17 April 1999; pp. 174-180.

91. Mohamad-Saleh, J.; Hoyle, B.S.; Podd, F.J.W.; Spink, D.M. Direct flow process estimations from tomographic data using artificial neural systems. In Proceedings of the SPIE 4188, Process Imaging for Automatic Control, Hannover, Germany, 2 February 2001; pp. 751-758. [CrossRef] 
92. Hua, L.; Dong, F.; Qiao, X.; Cui, X. Research on electrical resistance tomography and cross-correlation technique to measure the two-phase flows. In Proceedings of the Instrumentation and Measurement Technology Conference, Como, Italy, 18-20 May 2004; pp. 2308-2313. [CrossRef]

93. Tan, C.; Dong, F. Two-phase flow measurement by dual-plane ERT system with drift-flux model and cross-correlation technique. In Proceedings of the International Conference on Machine Learning and Cybernetics, Dalian, China, 13-16 August 2006; pp. 1443-1448. [CrossRef]

94. Stephenson, D.R.; Cooke, M.; Kowalski, A.; York, T.A. Investigating jet mixing using electrical resistance tomography. In Proceedings of the 5th International Symposium on Measurement Techniques for Multiphase Flows (5th ISMTMF), 2nd Int. Workshop on Process Tomography (IWPT-2) and 5th ISMTMF/IWPT-2, Macau, China, 10-13 December 2006; pp. 786-791. [CrossRef]

95. Xu, Y.; Wang, H.; Cui, Z.; Dong, F.; Yin, W. Cyclostationarity in electrical resistance tomography data from gas/liquid two-phase flow. In Proceedings of the Instrumentation and Measurement Technology Conference, Austin, TX, USA, 3-6 May 2010; pp. 259-263. [CrossRef]

96. Silva, R.; Garcia, F.; Faia, P.; Krochak, P.; Söderberg, D.; Lundell, F.; Rasteiro, G. Validating Dilute Settling Suspensions Numerical Data through MRI, UVP and EIT measurements. Flow Meas. Instrum. 2016, 50, 35-48. [CrossRef]

97. Zhao, X.; Lucas, G.P. Use of a novel dual-sensor probe array and electrical resistance tomography for characterization of the mean and time-dependent properties of inclined bubbly oil-in-water pipe flows. Meas. Sci. Technol. 2010, 22, 104001. [CrossRef]

98. Miettinen, T.; Laakhonen, M.; Aittamaa, J. Comparison of various flow visualisation techniques in a gas-liquid mixed tank. Comput. Aided Chem. Eng. 2003, 14, 773-778. [CrossRef]

99. Simmons, M.J.H.; Edwards, I.; Hall, J.F.; Fan, X.; Parker, D.J.; Stitt, E.H. Techniques for visualization of cavern boundaries in opaque industrial mixing systems. Aiche J. 2009, 55, 2765-2772. [CrossRef]

100. Stanley, S.J.; Mann, R.; Primrose, K. Tomographic imaging of fluid mixing in three dimensions for single-feed semi-batch operation of a stirred vessel. Chem. Eng. Res. Des. 2002, 80, 903-909. [CrossRef]

101. Mann, R. ERT imaging and linkage to CFD for stirred vessels in the chemical process industry. In Proceedings of the IEEE International Workshop on Imaging Systems and Techniques, Shenzhen, China, 11-12 May 2009; pp. 218-222. [CrossRef]

102. Stevenson, R.; Harrison, S.T.L.; Miles, N.; Cilliers, J.J. Examination of swirling flow using electrical resistance tomography. Powder Technol. 2006, 162, 157-165. [CrossRef]

103. Razzak, S.A.; Zhu, J.X.; Barghi, S. Effects of particle shape, density, and size on a distribution of phase holdups in a gas-liquid-solid circulating fluidized bed riser. Ind. Eng. Chem. Res. 2010, 49, 6998-7007. [CrossRef]

104. Harrison, S.T.L.; Stevenson, R.; Cilliers, J.J. Assessing solids concentration homogeneity in Rushton-agitated slurry reactors using electrical resistance tomography (ERT). Chem. Eng. Sci. 2012, 71, 392-399. [CrossRef]

105. Tahvildarian, P.; Ng, H.; D'Amato, M.; Drappel, S.; Ein-Mozaffari, F.; Upreti, S.R. Using electrical resistance tomography images to characterize the mixing of micron-sized polymeric particles in a slurry reactor. Chem. Eng. J. 2011, 172, 517-525. [CrossRef]

106. Razzak, S.A.; Zhu, J.X.; Barghi, S. Particle shape, density, and size effects on the distribution of phase holdups in an LSCFB riser. Chem. Eng. Technol. 2009, 32, 1236-1244. [CrossRef]

107. Xu, J.Y.; Wu, X.Z.; Zheng, C.; Wang, M.; Munir, B.; Oluwadarey, H.I.; Schlaberg, H.I.; Williams, R.A. Measurement of solid slurry flow via correlation of Electromagnetic Flowmeter, electrical resistance tomography and mechanistic modelling. J. Hydrodyn. 2009, 21, 557-563. [CrossRef]

108. Li, R.F.; Liu, L.; Wang, X.Z.; Tweedie, R.; Primrose, K.; Corbett, J.; McNeil-Watson, F. Multivariate statistical control of emulsion and nanoparticle slurry processes based on process tomography, dynamic light scattering, and acoustic sensor data. Comput. Aided Chem. Eng. 2009, 27, 1317-1322. [CrossRef]

109. Gumery, F.; Ein-Mozaffari, F.; Dahman, Y. Macromixing hydrodynamic study in draft-tube air lift reactors using electrical resistance tomography. Bioprocess. Biosyst. Eng. 2011, 34, 135-144. [CrossRef] [PubMed]

110. Kourunen, J.; Niitti, T.; Heikkinen, L.M. Application of three-dimensional electrical resistance tomography to characterize gas holdup distribution in laboratory flotation cell. Miner. Eng. 2011, 24, 1677-1686. [CrossRef]

111. Liu, L.; Li, R.F.; Collins, S.; Wang, X.Z.; Tweedie, R.; Primrose, K. Ultrasound spectroscopy and electrical resistance tomography for on line characterisation of concentrated emulsions in crossflow membrane emulsifications. Powder Technol. 2011, 213, 123-131. [CrossRef] 
112. Rasteiro, G.; Faia, P.; Garcia, F.; Santos, D. Oil/Water Stratified Flow in a Horizontal Pipe: Simulated and Experimental Studies using EIT. J. Pet. Sci. Eng. 2019, 174, 1179-1193. [CrossRef]

113. Rasteiro, G.; Faia, P.; Garcia, F.; Santos, D. Experimental and Simulated Studies of Oil/Water Fully Dispersed Flow in a Horizontal Pipe. J. Fluids Eng. 2019, 141. [CrossRef]

114. Henningsson, M.; Östergren, K.; Dejmek, P. Plug flow of yoghurt in piping as determined by cross-correlated dual-plane electrical resistance tomography. J. Food Eng. 2006, 76, 163-168. [CrossRef]

115. Regner, M.; Henningsson, M.; Wiklund, J.; Östergren, K.; Trägårdh, C. Predicting the displacement of Yoghurt by water in a pipe using CFD. Chem. Eng. Technol. 2007, 30, 844-853. [CrossRef]

116. Sharifi, M.; Young, B. 3-Dimensional spatial monitoring of tanks for the milk processing industry using electrical resistance tomography. J. Food Eng. 2011, 105, 312-319. [CrossRef]

117. Wang, M.; Lucas, G.; Dai, Y.; Panayotopoulos, N.; Williams, R.A. Visualisation of bubbly velocity distribution in a swirling flow using Electrical Resistance Tomography. Part. Part. Syst. Charact. 2006, 23, 321-329. [CrossRef]

118. Pakzad, L.; Ein-Mozaffari, F.; Upreti, S.R.; Lohi, A. Characterisation of the mixing of non-newtonian fluids with a scaba 6SRGT impeller through ERT and CFD. Can. J. Chem. Eng. 2013, 91, 90-100. [CrossRef]

119. Dong, F.; Qiao, X.T.; Jiang, Z.X.; Xu, L.A. Void fraction measurement of the gas-liquid two-phase flows in vertical pipes using electrical resistance tomography. J. Tianjin Univ. 2004, 37, 510-514.

120. Deng, X.; Li, G.; Wei, Z.; Yan, Z.; Yang, W. Theoretical study of vertical slug flow measurement by data fusion from electromagnetic flowmeter and electrical resistance tomography. Flow Meas. Instrum. 2011, 22, 272-278. [CrossRef]

121. Stanley, S.J.; Mann, R.; Primrose, K. Interrogation of a precipitation reaction by electrical resistance tomography (ERT). Aiche J. 2005, 51, 607-614. [CrossRef]

122. Vlaev, D.; Wang, M.; Dyakowski, T.; Mann, R.; Grieve, B.D. Detecting filter-cake pathologies in solid-liquid filtration: Semi-tech scale demonstrations using electrical resistance tomography (ERT). Chem. Eng. J. 2000, 77, 87-91. [CrossRef]

123. Yenjaichon, W.; Grace, J.R.; Lim, C.J.; Bennington, C.P.J. Gas dispersion in horizontal pulp-fibre-suspension flow. Int. J. Multiph. Flow 2013, 49, 49-57. [CrossRef]

124. Kourunen, J.; Heikkinen, L.M.; Paananen, P.; Peltonen, K.; Käyhkö, J.; Vauhkonen, M. Chemical pulping: Electrical resistance tomography for evaluating a medium consistency mixer. Nord. Pulp Pap. Res. J. 2011, 26, 179-185. [CrossRef]

125. Zhou, S.; Halttunen, J. Consistency Profile Measurement in Pulp Based on Electrical Impedance Tomography. In Proceedings of the XVII IMEKO World Congress Metrology in the 3rd Millennium, Dubrovnik, Croatia, 22-27 June 2003; pp. 22-27.

126. Ruzinsky, F.; Bennington, C.P.J. Aspects of liquor flow in a model chip digester measured using electrical resistance tomography. Chem. Eng. J. 2007, 130, 67-74. [CrossRef]

127. Hui, L.K.; Bennington, C.P.J.; Dumont, G.A. Cavern formation in pulp suspensions using side-entering axial-flow impellers. Chem. Eng. Sci. 2009, 64, 509-519. [CrossRef]

128. Cotas, C.; Branco, B.; Asendrych, D.; Garcia, F.; Faia, P.; Rasteiro, G. Experimental Study and Computational Fluid Dynamics Modelling of Pulp Suspensions Flow in a Pipe. J. Fluids Eng. 2017, 139, 071303. [CrossRef]

129. Ventura, C.; Garcia, F.A.P.; Ferreira, P.; Rasteiro, M.G. Flow Dynamics of Pulp Fibre Suspensions. Tappi J. 2008, 7, 20-26.

130. Polydorides, N.; Lionheart, W.R.B. A Matlab toolkit for three-dimensional electrical impedance tomography: A contribution to the Electrical Impedance and Diffuse Optical Reconstruction Software project. Meas. Sci. Technol. 2002, 13, 1871-1883. [CrossRef]

131. Cheng, K.S.; Isaacson, D.; Newell, J.C.; Gisser, D.G. Electrode models for electric current computed tomography. IEEE Trans. Biomed. Eng. 1989, 36, 918-924. [CrossRef]

132. Mosorov, V.; Sankowski, D.; Mazurkiewicz, L.; Dyakowski, T. The 'best-correlated pixels' method for solid mass flow measurements using electrical capacitance tomography. Meas. Sci. Tech. 2002, 13, 1810-1814. [CrossRef]

133. Dong, F.; Xu, Y.B.; Xu, L.J.; Hua, L.; Qiao, X.T. Application of dual-plane ERT system and cross-correlation technique to measure gas-liquid flows in vertical upward pipe. Flow Meas. Instrum. 2005, 16, 191-197. [CrossRef]

(C) 2020 by the authors. Licensee MDPI, Basel, Switzerland. This article is an open access article distributed under the terms and conditions of the Creative Commons Attribution (CC BY) license (http://creativecommons.org/licenses/by/4.0/). 